\title{
Initial Model for the Impact of Social Distancing on CoVID-19 Spread
}

\author{
Genghmun Eng \\ PhD Physics 1978, University of Illinois at Urbana-Champaign
}

April 12, 2020, updated April 21, 2020

\begin{abstract}
The initial stages of the CoVID-19 coronavirus pandemic all around the world exhibit a nearly exponential rise in the number of infections with time. Planners, governments, and agencies are scrambling to figure out "How much? How bad?" and how to effectively treat the potentially large numbers of simultaneously sick people. Modeling the CoVID-19 pandemic using an exponential rise implicitly assumes a nearly unlimited population of uninfected persons ("dilute pandemic"). Once a significant fraction of the population is infected ("saturated pandemic"), an exponential growth no longer applies. A new model is developed here, which modifies the standard exponential growth function to account for factors such as Social Distancing. A Social Mitigation Parameter [SMP] $\alpha_{S}$ is introduced to account for these types of society-wide changes, which can modify the standard exponential growth function, as follows:

$$
N(t)=N_{o} \exp \left[+K_{o} t /\left(1+\alpha_{S} t\right)\right]
$$

The doubling-time $t_{d b l}=(\ln 2) / K_{o}$ can also be used to substitute for $K_{o}$, giving a $\left\{t_{d b l}, \alpha_{S}\right\}$ parameter pair for comparing to actual CoVID-19 data. This model shows how the number of CoVID-19 infections can self-limit before reaching a saturated pandemic level. It also provides estimates for: (a) the timing of the pandemic peak, (b) the maximum number of new daily cases that would be expected, and (c) the expected total number of CoVID-19 cases. This model shows fairly good agreement with the presently available CoVID-19 pandemic data for several individual States, and the for the USA as a whole (6 Figures), as well as for various countries around the World ( 9 Figures). An augmented model with two Mitigation Parameters, $\alpha_{S}$ and $\beta_{S}$, is also developed, which can give better agreement with the daily new CoVID-19 data. Data-to-model comparisons also indicate that using $\alpha_{S}$ by itself likely provides a worstcase estimate, while using both $\alpha_{S}$ and $\beta_{S}$ likely provides a best-case estimate for the CoVID-19 spread.
\end{abstract}




\section{Introduction}

The Coronavirus 2019 disease (CoVID-19), caused by the SARS-CoV-2 (Severe Acute Respiratory Syndrome Coronavirus 2) pathogen, is now a world-wide pandemic. In many localities, the number of cases $N(t)$ was found to have an initial period of nearly exponential growth:

$$
\begin{aligned}
& N(t)=N_{o} \exp \left[+K_{D} t\right], \\
& K_{D} \equiv(\ln 2) / t_{D},
\end{aligned}
$$

aside of the first few cases, which may be untraceable. In Eqs. [1.1a]-[1.1b], $N_{o}$ is the initial number of infections at the $t=0$ start of data tracking, $K_{D}$ is an exponential growth factor, and $t_{D}$ is the doubling-time. Each locality can have its own $\left\{N_{o}, t_{D}, t=0\right\}$ values, and $K_{D}$ and $t_{D}$ should be nearly constant during this initial period of CoVID-19 spread.

Standard epidemiology identifies the number of people $N_{G}$ a known infected individual had recent contact with. Contacts of that $N_{G}$ group are tracked next, followed by additional tracking stages. This process sets the $K_{D}$ value.

Society-wide Mitigation Measures such as: (a) Social Distancing, (b) wearing face masks in public, (c) prohibiting large gatherings, (d) implementing largescale population testing, (e) disinfecting high-touch surfaces in public areas, (f) enhanced cleaning of items brought into homes, and (g) minimizing human contact with likely virus-containing materials and matter; all can help reduce $N(t)$ growth. These Mitigation Measures can modify the Eq. [1.1a] epidemiology model by causing the local $t_{D}$ values to lengthen.

In order to model these Mitigation Measures, $t_{D}$ and $K_{D}$ become explicit functions of time, $t_{D}(t)$ and $K_{D}(t)$. Using a linear function for $t_{D}(t)$ lengthening is one of the simplest time-varying extensions. A linear function of time also corresponds to the first term of a Taylors' Series expansion of some more general $t_{D}(t)$ analytic function, giving this epidemiology extension:

$$
\begin{aligned}
& t_{D}(t) \equiv t_{d b l}\left(1+\alpha_{S} t\right), \\
& K_{D}(t) \equiv \frac{(\ln 2)}{t_{D}(t)}=(\ln 2) /\left[t_{d b l}\left(1+\alpha_{S} t\right)\right] \equiv K_{o} /\left(1+\alpha_{S} t\right) .
\end{aligned}
$$

The $t=0$ initial values for $t_{D}(t)$ and $K_{D}(t)$ become the new constants $t_{d b l}$ and $K_{o}$, which characterize the initial exponential growth phase. The $\alpha_{S}$ coefficient in Eq. [1.3a] is a new Social Mitigation Parameter [SMP] that helps quantify the effectiveness of the society-wide Mitigation Measures as a whole.

The $\alpha_{S}$ value expresses how well non-infected people manage to avoid the virus contagion. As a lumped parameter, it likely reflects an average value over many processes, known and unknown, which comprise mitigation, to supplement the contact-to-contact tracking that initially sets $t_{d b l}$ or $K_{o}$.

Substituting Eq. [1.3b] into Eq. [1.1a] gives:

$$
N(t)=N_{o} \exp \left[+K_{o} t /\left(1+\alpha_{S} t\right)\right],
$$

as one of the simplest models for CoVID-19 spread. A pure exponential growth (or decay) has no memory, while Eq. [1.4], for $\alpha_{S}>0$, has a memory. The $t=0$ start time of first mitigation changes the future history. To include $t<0$ requires replacing Eq. [1.3a] by $t_{D}(t) \equiv t_{d b l}\left(1+\max \left[0, \alpha_{S} t\right]\right)$, which has a corner at $t=0$ that preserves the memory of when mitigation first started. 


\section{Model Features}

The Eq. [1.1a] exponential growth pandemic model implicitly assumes a large uninfected population allows the disease to easily spread ("dilute pandemic"). When almost everybody is infected ("saturated pandemic"), exponential growth shuts off, and Eq. [1.1a] no longer applies.

On 3/10/2020, German Chancellor Angela Merkel ${ }^{1}$ noted that she "estimates that $60 \%$ to $70 \%$ of the German population will contract the coronavirus", indicating that saturated pandemic models are being considered as a worst-case.

Even that worst-case condition assumes: (i) recovered coronavirus patients are no longer infectious, and (ii) surviving a CoVID-19 infection confers absolute immunity to re-infection. Recently, South Korea ${ }^{2}$ found 91 cases of clinically recovered patients later testing as CoVID-19 positive. They may also shed viable coronaviruses in their phlegm and fecal matter ${ }^{3}$, furthering disease spread. Although these effects are not modeled here, those additional transmission modes could turn a $60 \%-70 \%$ hope into a $99+\%$ consequence.

These factors show why CoVID-19 modeling beyond Eq. [1.1] is needed, especially to see if society-wide Mitigation Measures can naturally halt disease spread, without necessitating a saturated pandemic condition. We show next that Eq. [1.4] allows for this pandemic shut-off, even in the dilute pandemic case. Since both $K_{o}$ and $\alpha_{S}$ in Eq. [1.4] have the same units, their ratio is dimensionless. The long-term limit of Eq. [1.4] gives:

$$
\operatorname{Lim}_{t \rightarrow+\infty}\left[N(t) / N_{o}\right]=\exp \left[+K_{o} / \alpha_{S}\right]
$$

setting an average value for the total number of all follow-on infections arising from a single individual. Since it depends only on the ratio of the original pandemic growth factor $K_{o}$ to the $\alpha_{S} S M P$, this model shows the impact of accounting for a broader environment beyond individual contact tracking.

The early spread of CoVID-19 cases outside of China ${ }^{4}$, and the early USA CoVID-19 data ${ }^{\mathbf{5}}$ both had nearly exponential rises, as shown in Figure 1. A purely exponential rise gives a straight line on a $\log$-plot $\{\log (\#$ of cases) vs linear time $\}$. The initial doubling-time for the USA was $t_{d b l} \approx 2.02$ days, giving $K_{o} \approx 0.343 /$ day using Eqs. [1.1a]-[1.1b]. This initial CoVID-19 data was prior to any significant Mitigation Measures being implemented.

On March 19, 2020, Governor Gavin Newsom of California ordered a CoVID19 "stay-at-home" lockdown of virtually all of California's $\sim 40$ million residents. Similar statewide CoVID-19 lockdowns were ordered by the Governors of Illinois, New York, Indiana, Michigan, Ohio, Washington, West Virginia and Wisconsin.

The slowing of CoVID-19 spread by implementing large-scale societal Mitigation Measures can be fairly rapid, as illustrated by the USA CoVID-19 data of Figure 2, which covers March 2020.

The impact of these multi-state Mitigation Measures is evident in Figure 2 as a sudden transition on the log-plot from a straight-line to having downward curvature, which the $\alpha_{S}$ Social Mitigation Parameter (SMP) aims to quantify. The local slope in Figure 2 also decreases right after the onset of Mitigation Measures, indicating further slowing of CoVID-19 spread. 
A well-documented South Korean coronavirus cluster can also be used to help estimate the expected size of the $\alpha_{S} S M P$. That CoVID-19 cluster determined that a single infected person at the Shinjeongji Church caused infection of about 4,482 people $^{4}$ within the 47-day time interval between January 20, 2020 and March 8, 2020. Those data provide this $\alpha_{S}$ estimate:

$$
\begin{array}{ll}
\ln (4,482) \approx(0.343 / \text { day })(47 \text { days }) /\left[1+\alpha_{S}(47 \text { days })\right], & {[2.2 \mathrm{a}]} \\
\alpha_{S}=0.01952 / \text { day } \approx 0.02 / \text { day } & {[2.2 \mathrm{~b}]}
\end{array}
$$

as indicative of minimal mitigation. If additional deliberate mitigation measures doubled $\alpha_{S}$ to $\alpha_{S} \approx(0.04 /$ day $)$, Eq. [2.1] would give:

$$
\operatorname{Lim}_{t \rightarrow+\infty}\left[N(t) / N_{o}\right]=\exp \left[+\frac{(0.343 / \text { day })(47 \text { days })}{1+(.04 / \text { day })(47 \text { days })}\right] \approx 270,
$$

for the number of infections per person, a $16.6 \mathrm{X}$ reduction from 4,482 .

Since $N(t)$ in Eq. [1.4] represents a total number of cases, it is similar to a cumulative distribution function (cdf), which is used in reliability and also has time as its fundamental variable. The derivative of Eq. [1.4], $d N(t) / d t$, is analogous to an unnormalized probability density function ( $p d f$ ), which can be used to predict a pandemic peak:

$$
\begin{aligned}
& \{p d f\} \equiv \frac{d}{d t}\left[\frac{N(t)}{N_{o}}\right]=\frac{d}{d t}\left\{\exp \left[+K_{o} t /\left(1+\alpha_{S} t\right)\right]\right\}= \\
& +K_{o}\left(\frac{1}{1+\alpha_{S} t}\right)^{2} \exp \left[+K_{o} t /\left(1+\alpha_{S} t\right)\right] \\
& \{p d f\}=+K_{o}\left[\frac{N(t)}{N_{o}}\right] /\left(1+\alpha_{S} t\right)^{2}
\end{aligned}
$$

The time $t_{P}$ of the pandemic peak is set by:

$$
\begin{aligned}
& \frac{d}{d t}\{p d f\} \equiv 0, \\
& \alpha_{S} t_{P}=\frac{1}{2}\left[K_{o} / \alpha_{S}\right]-1,
\end{aligned}
$$

where Eq. [2.5b] simplification arises from the Eq. [2.5a] constraint. Substituting $K_{o}=0.343 /$ day and $\alpha_{S}=0.02 /$ day from Eq. [2.2b] into Eq. [2.5b] gives $t_{P} \approx 379$ days. Increasing mitigation to $\alpha_{S}=0.04 /$ day, keeping the same $K_{o}=0.343 /$ day, now gives $t_{P} \approx 82$ days, which is a $\sim 4.6 X$ reduction in the pandemic peak timing for doubling $(2 X)$ the mitigation effect from its original baseline value. These examples highlight the tremendous impact that even a small amount of enhanced social mitigation can have.

While the $\alpha_{S}=0$ limit of Eq. [2.4b] recovers the Eq. [1.1a] standard exponential growth, both the $\{p d f\}$ and $\left[N(t) / N_{o}\right]$ growth are then unbounded. However, even a small $\alpha_{S}>0$ value in Eq. [2.4b] will have an enormous impact on the predicted long-time behavior. Since Eq. [2.1] showed that $\left[N(t) / N_{o}\right]$ now approaches a finite value for all $\alpha_{S}>0$, this new $\{p d f\}$ asymptotic limit:

$$
\operatorname{Lim}_{t \rightarrow+\infty, \alpha_{S}>0}[\{p d f\}] \sim \frac{\{\text { Constant }\}}{\left(\alpha_{S} t\right)^{2}},
$$

also results. The $\{p d f\}$ that arises from this model all have an initial exponential rise, coupled with the Eq. [2.6] "long tail" at large times, which means that new CoVID-19 cases may arise for a long time, even if significant Mitigation Measures are in place.

The Eq. [2.6] $\{p d f\}$ prediction also differs substantially from the widely-used University of Washington IHME (Institute for Health Metrics and Evaluation) projections, which use symmetric Gaussians for both the $\{p d f\}$ rise and fall ${ }^{\mathbf{6}}$. Thus, these methods provide an alternative risk-bound for evaluating potential CoVID-19 worst-case scenarios. 


\section{Determining $\left\{t_{d b l}, \alpha_{S}\right\}$ from CoVID-19 Data}

Explicit numerical values for $\left\{t_{d b l}, \alpha_{S}\right\}$ parameters were determined from the CoVID-19 data as follows. Rewriting Eq. [1.4] as:

$$
\ln \left[N(t) / N_{o}\right]=\left[+K_{o} t /\left(1+\alpha_{S} t\right)\right] \equiv(\ln 2) t /\left[t_{d b l}\left(1+\alpha_{S} t\right)\right] .
$$

allowed data fitting to be done on a $Y-v s-X$ log-plot, using $Y=$ $\ln \left[N(t) / N_{o}\right]$ as the ordinate and $X \leftrightarrow t$ as the abscissa, to calculate and minimize the root-mean-square (rms) error.

The $t=0$ point in Eq. [3.1] sets $N_{o}$. To best model Mitigation Measures, this point was usually chosen at the start of a downward curvature on a log-plot, so that $N(t=0) \equiv N_{I}$, where the $N_{I}$ is now the first data point in the analysis. The prior $t<0$ regime can often have a nearly pure exponential growth, as in Figure 2, and those regions should not be part of rms-error minimization for evaluating Mitigation Measures.

The $N_{F}$ final data point, measured at the most recent $t=t_{F}$ time:

$$
N\left(t=t_{F}\right) \equiv N_{F} \text {. }
$$

was also fixed for each dataset, so that only $\left\{t_{d b l}, \alpha_{S}\right\}$ value pairs that meet both $N(t=0) \equiv N_{I}$ and Eq. [3.2] were used.

In practice, an $\alpha_{S}$ was chosen first. The Excel ${ }^{T M}$ _Tools_Goal-Seek function was used to adjust $t_{d b l}$ to obey Eq. [3.2], setting the $r \bar{m} s$-error between the dataset and Eq. [3.1], with the final $\left\{t_{d b l}, \alpha_{S}\right\}$ having the least rms-error.

In the following figures, all CoVID-19 raw data came from the publicly available Microsoft ${ }^{\mathrm{TM}}$ "COVID-19 Tracker" site ${ }^{7}$. When no updates were available, that site repeated the prior day data, whereas we used the geometric mean of the day-prior and day-after data for interpolation.

\section{Effects of Varying the Initial Zero-Time Point}

Starting with:

$$
\begin{aligned}
& N(t)=\mathbf{1} \exp \left[+K_{o} t /\left(1+\alpha_{S} t\right)+\ln \left(N_{o}\right)\right], \\
& \operatorname{Lim}[N(t)]=\mathbf{1} \exp \left[+\left(K_{o} / \alpha_{S}\right)+\ln \left(N_{o}\right)\right], \\
& t \rightarrow+\infty \\
& t_{d b l}^{o} \equiv(\ln 2) / K_{o},
\end{aligned}
$$

using a shifted time-scale normalization point is examined next:

$$
N_{S}\left(t^{\prime}\right)=1 \exp \left[+K_{o}\left(t^{\prime}+t_{A}\right) /\left[1+\alpha_{S}\left(t^{\prime}+t_{A}\right)\right]+\ln \left(N_{o}\right)\right], \quad[4.2]
$$

This $N_{S}\left(t^{\prime}\right)$ function should closely match Eq. [4.1a], with a shifted time axis: $t=t^{\prime}+t_{A}$, but the best fit parameter numerical values change. Since:

then defining:

$$
\begin{aligned}
& {\left[1+\alpha_{S}\left(t^{\prime}+t_{A}\right)\right]=\left[1+\alpha_{S} t_{A}\right]\left[1+\frac{\alpha_{S} t^{\prime}}{\left(1+\alpha_{S} t_{A}\right)}\right],} \\
& \frac{K_{o}\left(t^{\prime}+t_{A}\right)}{\left[1+\alpha_{S}\left(t^{\prime}+t_{A}\right)\right]}=\left[1+\frac{t_{A}}{t^{\prime}}\right] \frac{\left(K_{o} t^{\prime}\right)}{\left[1+\alpha_{S} t_{A}\right]} /\left[1+\frac{\alpha_{S} t^{\prime}}{\left(1+\alpha_{S} t_{A}\right)}\right],
\end{aligned}
$$

$$
\begin{array}{lr}
\alpha_{A} \equiv \alpha_{S} /\left(1+\alpha_{S} t_{A}\right), & {[4.4 \mathrm{a}]} \\
K_{A} \equiv K_{o} /\left(1+\alpha_{S} t_{A}\right), & {[4.4 \mathrm{~b}]} \\
t_{d b l}^{A} \equiv(\ln 2) / K_{A}=\left(1+\alpha_{S} t_{A}\right) t_{d b l}^{o}=\left(1+\alpha_{S} t_{A}\right)(\ln 2) / K_{o},[4.4 \mathrm{c}]
\end{array}
$$

it gives:

$$
\frac{K_{o}\left(t^{\prime}+t_{A}\right)}{\left[1+\alpha_{S}\left(t^{\prime}+t_{A}\right)\right]}=\left[1+\frac{t_{A}}{t^{\prime}}\right] K_{A} t^{\prime} /\left[1+\alpha_{A} t^{\prime}\right]
$$




$$
=K_{A} t^{\prime} /\left[1+\alpha_{A} t^{\prime}\right]+K_{A} t_{A} /\left[1+\alpha_{A} t^{\prime}\right] .
$$

These equations highlight the net effect of time-shifting. For $t_{A}>0$, when $t^{\prime}$ begins after Mitigation Measures have started, the shifted-time-axis results in a larger calculated doubling-time and a smaller $S M P \alpha_{A}$-value. For $t_{A}<0$, when $t^{\prime}$ may include Mitigation Measures already in place at the Eqs. [4.1a]-[4.1c] $t=0$ point, this shifted-time-axis results in a smaller calculated doubling-time and a larger $S M P \alpha_{A}$-value.

Finally, for small $t^{\prime}$, where $\alpha_{A} t^{\prime}<1$, using Eqs. [4.1b] and [4.5] gives:

$$
\begin{aligned}
& 1 \exp \left[\frac{+K_{o} t}{\left(1+\alpha_{S} t\right)}+\ln \left(N_{o}\right)\right] \approx 1 \exp \left[\frac{+K_{A} t^{\prime}}{\left(1+\alpha_{A} t^{\prime}\right)}+\ln \left(N_{A}\right)\right], \\
& \ln \left(N_{A}\right)=\ln \left(N_{o}\right)+K_{A} t_{A},
\end{aligned}
$$

which shows that the $t^{\prime}=0$ new initial state should have an $N_{A}$ starting value obeying $N_{A}>N_{o}$ for $t_{A}>0$, and $N_{A}<N_{o}$ for $t_{A}<0$. However, whether $\left\{N_{o}, K_{o}, \alpha_{S}\right\}$, or an alternative $\left\{N_{A}, K_{A}, \alpha_{A}\right\}$, are used to parameterize a given data set, the net overall function fit and predictions, as a function of calendar date, should remain fairly self-consistent, even when some ambiguity exists as to when Mitigation Measures first were noticeably effective.

\section{$5 \quad$ USA and Selected States Model Results}

The model predictions for CoVID-19 spread in the USA is shown in Figure 3. This analysis only included data after mid-March 2020, when several State Governors first instituted mandatory Mitigation Measures. Results give an $S M P$ estimate of $\alpha_{S} \approx 0.5945 /$ day, a USA initial doubling-time of $t_{d b l}^{\text {initial }} \approx 2.1758$ days, which lengthens to $t_{d b l}^{\{a t P e a k\}} \approx 5.83$ days at the projected pandemic peak of $\sim 4 / 19 / 2020$. The predicted total number of CoVID-19 cases is $\sim 5,464,000$, giving a projected $\sim 1.67 \%$ final infection rate, if the present level of Mitigation Measures or their equivalent, are continued.

These predictions assume no "second wave" of infection or re-infection. They also do not include the effect of additional Mitigation Measures, which could further increase the $\left\{t_{d b l}, \alpha_{S}\right\}$ values, and significantly reduce the projected final number of CoVID-19 cases.

Figure 4 shows model predictions for CoVID-19 evolution in California. Only data after 3/21/2020 was included in the analysis, after California Governor Gavin Newsom instituted mandatory Mitigation Measures. It gives an SMP estimate of $\alpha_{S} \approx 0.03546 / d a y$, with an initial doubling-time of $t_{\text {dbl }}^{\text {initial }} \approx$ 2.5017 days, which lengthens to $t_{d b l}^{\{a t P e a k\}} \approx 9.774$ days at the projected pandemic peak of $\sim 6 / 07 / 2020$. The predicted total number of CoVID-19 cases is $\sim 1,123,700$, giving a projected $\sim 2.813 \%$ final infection rate, at the present level of Mitigation Measures.

Figure 5 shows model predictions for CoVID-19 evolution in New York. A relatively high $S M P$ estimate of $\alpha_{S} \approx 0.1031 /$ day was found, coupled with a relatively short initial doubling-time of $t_{\text {dbl }}^{\text {initial }} \approx 0.9395$ days, which creates a high narrow spike in daily new cases. The present model projects a New York pandemic peak around 4/10/2020, with an estimated at-peak doubling-time of 
$t_{d b l}^{\{a t \text { Peak }\}} \approx 3.36$ days. The predicted total number of cases at $\sim 1,218,000$, giving a projected $\sim 6.072 \%$ final infection rate.

Figure 6 shows model predictions for CoVID-19 evolution in Washington State. An initial doubling-time of $t_{d b l}^{\text {initial }} \approx 2.189$ days and an $S M P$ value of $\alpha_{S} \approx 0.0687 /$ day were found, with a projected pandemic peak around 6/04/2020. The relatively low number of cases at the Mitigation Measures start helps to give a predicted total number of cases of $\sim 557,600$, corresponding to a $7.15 \%$ final infection rate.

Figure 7 shows model predictions for CoVID-19 evolution in Illinois. An initial doubling-time of $t_{d b l}^{\text {initial }} \approx 2.457$ days and moderate $S M P$ value of $\alpha_{S} \approx 0.0373 /$ day combine to give a projected pandemic peak around 6/04/2020, similar to Washington State, but having a higher predicted total number of cases at $\sim 1,277,000$, and a projected $\sim 11.47 \%$ final infection rate.

Figure 8 shows model predictions for CoVID-19 evolution in Florida. Many Florida counties instituted their own Mitigation Measures prior to a state-wide lockdown, slowing CoVID-19 growth. A somewhat high SMP value of $\alpha_{S} \approx$ $0.0526 /$ day, and an initial doubling-time of $t_{d b l}^{\text {initial }} \approx 1.494$ days results. A pandemic peak is estimated at around $5 / 20 / 2020$, with a predicted total number of cases at $\sim 1,090,000$, and a projected $\sim 4.96 \%$ final infection rate.

\section{World and Selected Countries Model Results}

Figure 9 shows model predictions of CoVID-19 evolution for the whole World. The present-day doubling-time value of $t_{\text {dbl }}^{\text {initial }} \approx 5.761$ days likely represents a combination of small urban, large urban, and rural area results. However, the calculated low $S M P$ estimate of $\alpha_{S} \approx 0.01712 /$ day shows that nearly $\sim 4.43 \%$ of the World's population could be at risk for eventual CoVID-19 infection. At these present levels, the projected pandemic peak is around 8/15/2020, with potentially hundreds of millions of people being infected.

Figure 10 shows model predictions for CoVID-19 evolution in China, covering their "first wave" of early exposure and early mitigation. Data were included that was prior to a "New Reporting Method" being used, which started off with one sudden data jump, and nearly level CoVID-19 follow-on results. The present model predicts what number of cases could have resulted, had the reporting method not changed. Draconian Mitigation Measures helped to contain the pandemic to Hubei Province and Wuhan. These projections show that those Mitigation Measures have impressively contained CoVID-19 spread.

Figure 11 shows model predictions for CoVID-19 evolution in South Korea, covering the period of their country's early exposure and initial mitigation methods. Pre-pandemic Mitigation Measures, including extensive contact-tracing and large-scale CoVID-19 testing, were implemented. These projections show that those Mitigation Measures, as an alternative to China's methods, also have impressively contained CoVID-19 spread.

Figure 12 shows model predictions for CoVID-19 evolution in Italy. An initial doubling-time of $t_{d b l}^{\text {initial }} \approx 1.4648$ days and SMP estimate of $\alpha_{S} \approx$ 
0.05282 /day give a pandemic peak around 4/29/2020, with a predicted number of total cases at $\sim 1,764,000$, and a projected $\sim 2.92 \%$ final infection rate.

Figure 13 shows model predictions for CoVID-19 evolution in Germany. The relatively high $S M P$ estimate of $\alpha_{S} \approx 0.07614 /$ day with an initial doublingtime of $t_{\text {dbl }}^{\text {initial }} \approx 1.4177$ days combine to give a projected pandemic peak at around 4/08/2020, with a predicted total number of cases of $\sim 700,100$, and a projected $0.84 \%$ final infection rate. These values would make Germany one of the less impacted countries in Europe. They represent predicted final CoVID19 infection rates that are significantly lower than the original $60 \%-70 \%$ early worst-case estimates highlighted by German Chancellor Angela Merkel.

Figure 14 shows model predictions for CoVID-19 evolution in Spain. An $S M P$ estimate of $\alpha_{S} \approx 0.07058 /$ day, which is comparable to Germany, and a smaller initial doubling-time of $t_{d b l}^{\text {initial }} \approx 1.1778$ days combine to give more predicted CoVID-19 cases than Germany. The estimated pandemic peak is around 4/21/2020, with a predicted number of total cases at $\sim 1,526,000$, and a projected $\sim 3.26 \%$ final infection rate.

Figure 15 shows model predictions for CoVID-19 evolution in Ecuador. Reports of chaos in Ecuador have been alarming. Yet the present data show a significant and somewhat unexpected leveling off in the number of reported CoVID-19 cases. This result could mean that some as yet unknown Mitigation Measures may be operating. Alternatively, the data could mean that there is a dire CoVID-19 testing and reporting shortfall operating amidst the chaos.

Figure 16 shows model predictions for CoVID-19 evolution in India. These initial data show virtually no mitigation at present, having one of the lowest calculated $S M P$ estimates of $\alpha_{S} \approx 0.0148 /$ day, with an initial doubling-time of $t_{d b l}^{\text {initial }} \approx 3.135$ days. At this rate, nearly $17.38 \%$ of the population of India could eventually become infected. The estimated pandemic peak is around $5 / 30 / 2021$, which would be 441 days after the first CoVID-19 fatality was reported, on 3/14/2020. Additional Mitigation Measures, further increasing the $\left\{t_{d b l}, \alpha_{S}\right\}$ values, as well as adding in additional modeling parameters may significantly reduce these projected number of CoVID-19 cases.

\section{Augmented Peak Shape Modeling}

Using a new Social Mitigation Parameter [SMP] $\alpha_{S}$, as in Eq. [1.3a], successfully models pandemic shut-off, even in the dilute pandemic limit. However, as the Figures 3-16 insets show, many of the data-vs-model comparisons have the data trending above the model near the final $t=t_{F}$ data point.

Since Eq. [1.3a] for $t_{D}(t)$ is linear, using an Additional Modeling Parameter $[A M P] \beta_{S}$ in a higher order polynomial, may fit the $\{p d f\}$ shape better. A quadratic function for $t_{D}$ :

$$
t_{D}=t_{d b l}\left(1-\beta_{S} Z+\alpha_{S} Z^{2}\right),
$$

where $N(t) / N_{o}$ still approaches a constant at long times, as in Eq. [2.1a], then sets $Z^{2} \equiv t$, giving this extension of Eq. [1.3a]:

$$
t_{D}(t)=t_{d b l}\left(1-\beta_{S} \sqrt{t}+\alpha_{S} t\right) \text {. }
$$


Values of $\beta_{S}>0$ in Eq. [5.2] allow the predicted $\left[N(t) / N_{o}\right]$ values to rise above the $\beta_{S}=0$ model predictions, and to have a smaller doubling-time, for the same $\left\{t_{d b l}, \alpha_{S}\right\}$. However, the best fit $\left\{t_{d b l}, \alpha_{S}\right\}$ values will also differ between the $\beta_{S}>0$ and $\beta \equiv 0$ cases, so these changes are relative.

The new $\{p d f\}$ function for Eq. [5.2] is:

$$
\begin{gathered}
\{p d f\} \equiv \frac{d}{d t}\left[\frac{N(t)}{N_{o}}\right]=\frac{d}{d t}\left\{\exp \left[+K_{o} t /\left(1-\beta_{S} \sqrt{t}+\alpha_{S} t\right)\right]\right\}= \\
+K_{o}\left[\frac{N(t)}{N_{o}}\right]\left(1-\frac{1}{4} \beta_{S}^{2} t\right) /\left[\left(1+\alpha_{S} t\right)^{2}\left(1+\frac{1}{2} \beta_{S} \sqrt{t}\right)\right] .
\end{gathered}
$$

When $\{p d f\}=0$ in Eq. [5.3], it estimates an end-point for the pandemic at:

$$
t_{E N D}=\left(4 / \beta_{S}^{2}\right),
$$

while predicting this maximum number of pandemic cases at $t_{E N D}$ :

$$
N(t)=N_{o} \exp \left[K_{o} /\left(\alpha_{S}-\frac{1}{4} \beta_{S}^{2}\right)\right] .
$$

An an example, this augmented model was applied to CoVID-19 evolution in Italy. As shown in Figure 17, this Eq. [5.3] $\{p d f\}$ function gives a better fit to the observed number of daily new CoVID-19 cases.

The initial doubling-time of $t_{d b l}^{\text {initial }} \approx 2.5566$ days, along with estimates for the Mitigation Measure parameters of $\alpha_{S} \approx 0.04583 /$ day and $\beta_{S} \approx-0.1725$, in this augmented model, combine to significantly reduce the projected maximum number of CoVID-19 cases down to $\sim 264,820$, which is an $\sim 7 X$ less compared to using $\alpha_{S}$ alone, as in Figure 12. This augmented model sets an estimated pandemic peak at $3 / 29 / 2020$, with a projected pandemic end-point around $7 / 7 / 2020$, which is also significantly more optimistic.

The true CoVID-19 pandemic progress is likely to be in between Figure 12 as a worst-case, and Figure $\mathbf{1 7}$ as a best-case projection. The geometric mean of the Figure 12 and Figure 17 results set an average of $~ 683,500$ cases for Italy at the CoVID-19 pandemic end. These bounds also highlight the amount of uncertainty that is intrinsic to these empirically based methods.

\section{Summary and Conclusions}

The standard exponential for modeling pandemics starts with an $N_{o}$ known number of initial cases at some reference time $t=0$. Epidemiologists work to determine a pandemic growth factor $K_{D}$, which sets the doubling-time $t_{D}$ for the number of pandemic cases.

The CoVID-19 disease, caused by the SARS-CoV-2 coronavirus pathogen, initially showed both regional and global exponential growth. It resulted in a doubling-time of $t_{D} \approx 2.02$ days for the US, as highlighted in Figure 1.

An exponential growth normally only halts when it runs out of materials. In epidemiology that point often occurs when there are virtually no more uninfected people left, which we call a saturated pandemic. The exponential growth function is only applicable when infection rates are much lower than saturation, which we call a dilute pandemic.

A modification to exponential growth is developed here, which allows ratio of the number of pandemic cases, $N(t)$, compared to its $N_{o}$ initial value at $t=0$ :

$$
\operatorname{Lim}_{t \rightarrow+\infty}\left[N(t) / N_{o}\right]=\mathbf{M},
$$


to approach a final constant, denoted $\mathbf{M}$, while still being in a dilute pandemic condition. This result is attributed to the inclusion of society-wide Mitigation Measures to stop pandemic growth, before the value of $\mathbf{M}$ reaches the whole population value.

Society-wide Mitigation Measures aim to progressively lengthen the $t_{D}$ doublingtime, essentially making $t_{D}(t)$. Most analyses presented here used a linear function of time as a simplest non-constant model for $t_{D}(t)$ :

$$
\begin{array}{ll}
t_{D}(t) \equiv t_{d b l}\left(1+\alpha_{S} t\right), & {[6.2 \mathrm{a}]} \\
K_{D}(t) \equiv \frac{(\ln 2)}{t_{D}(t)}=(\ln 2) /\left[t_{d b l}\left(1+\alpha_{S} t\right)\right] \equiv K_{o} /\left(1+\alpha_{S} t\right), & {[6.2 \mathrm{~b}]}
\end{array}
$$

where $t_{D}(t=0) \equiv t_{d b l}$, and $K_{D}(t=0) \equiv K_{o}$. Here, $\alpha_{S}$ is a new Social Mitigation Parameter (SMP), to quantify societal Mitigation Measures. This Eq. [6.2a] extension of a pure exponential growth gives:

$$
N(t)=N_{o} \exp \left[+K_{o} t /\left(1+\alpha_{S} t\right)\right]
$$

as an empirical equation for modeling CoVID-19 spread. Since both $K_{o}$ and $\alpha_{S}$ in Eq. [1.4] have the same units, their ratio is a dimensionless number. The long-term limit of Eq. [6.3] gives:

$$
\operatorname{Lim}_{t \rightarrow+\infty}\left[N(t) / N_{o}\right]=\exp \left[+K_{o} / \alpha_{S}\right]
$$

setting a final value for the Eq. [6.1] constant $\mathbf{M}$, allowing these predictions to be applicable to the dilute pandemic limit. The CoVID-19 number of estimated cases per day is given by:

$$
\begin{aligned}
& \{p d f\}=\frac{d}{d t}\left[N(t) / N_{o}\right], \\
& \quad \operatorname{Lim}_{t \rightarrow+\infty, \alpha_{S}>0}[\{p d f\}] \sim \frac{\{\text { Constant }\}}{\left(\alpha_{S} t\right)^{2}},
\end{aligned}
$$

which combines an initial exponential rise with "long tail" at large times. In this model, new CoVID-19 cases can continue to arise for a long time, even with significant Mitigation Measures in place.

Analysis of available CoVID-19 data using this model shows that it can match observed data fairly well, both from various US states [Figures 3-8], as well as for different global countries [Figures 9-17]. However, using a single parameter to encompass all societal Mitigation Measures often gives a slightly larger slope on a log-plot, compared to the latest measured data values, which makes this model a likely worst-case estimate.

A second data-fitting parameter $\beta_{S}$ was also used in an augmented model, to better fit the $\{p d f\}$ data:

$$
\begin{array}{ll}
N(t)=N_{o} \exp \left[+K_{o} t /\left(1-\beta_{S} \sqrt{t}+\alpha_{S} t\right)\right], & {[6.6 \mathrm{a}]} \\
\max \left[N(t) / N_{o}\right]=\exp \left[+K_{o} /\left(\alpha_{S}-\frac{1}{4} \beta_{S}^{2}\right)\right], & {[6.6 \mathrm{~b}]} \\
t_{E N D}=\left(4 / \beta_{S}^{2}\right), & {[6.6 \mathrm{c}]}
\end{array}
$$

where $t_{E N D}$ becomes an estimated pandemic end-point, where zero new CoVID-19 cases per day could occur.

As a representative example, this augmented model was applied to the CoVID-19 data from Italy [Figure 17]. Those results show that this augmented model allows a better fit to the observed number of new daily CoVID-19 cases, but the absence of a CoVID-19 tail in its $\{p d f\}$ function makes this $\left\{K_{o}, \alpha_{S}, \beta_{S}\right\}$ augmented model a likely best-case result, with the original $\left\{K_{o}, \alpha_{S}\right\}$ model being a likely worst-case estimate. 
This class of CoVID-19 pandemic models all enable pandemic shut-off even in the dilute pandemic limit, with only a small fraction of the total population being infected. These models also provide estimates for: (a) the maximum number of cases near pandemic shutoff, (b) the size and shape of the pandemic peak $[d N(t) / d t]$, and $(\mathrm{c})$ pandemic peak timing $\left[t_{P}\right]$. These models and analyses may help enhance planning and preparation to maximize resource use, potentially increasing individual and collective CoVID-19 pandemic survival rates.

\section{List of Figures}

Figure 1: Early CoVID-19 Cases: (a) Outside of China, and (b) Just in the US. Both graphs show nearly exponential growth.

Figure 2: USA CoVID-19 data, pre-vs-post mid-March 2020. Multi-State Mitigation Measures slowed growth, transitioning from straight-line to downward curvature.

Figure 3: USA Model Predictions. To allow better mitigation predictions, only data after mid-March 2020 was included, when several Governors instituted mandatory lockdowns.

Figure 4: Predicted California CoVID-19 results. After Gov. Gavin Newsom instituted widespread Mitigation Measures, projections showed significant improvement.

Figure 5: Predicted New York CoVID-19 results. A relatively high Mitigation Measure level and a short intrinsic doubling time creates a narrow spike in daily new cases.

Figure 6: Predicted Washington State CoVID-19 results. The relatively low number of cases at Mitigation Measure start helps to give a relatively low final number of cases.

Figure 7: Predicted Illinois CoVID-19 results. The slow doubling-time and moderate amount of Mitigation Measures gives a slow increase to the predicted CoVID-19 peak.

Figure 8: Predicted Florida CoVID-19 results. Many Florida counties instituted their own Mitigation Measures prior to a state-wide lockdown, substantially slowing CoVID-19 growth.

Figure 9: Model predictions for the WORLD, showing present-day low level of mitigation.

Figure 10: Predicted CHINA CoVID-19 results, using pre-"New Reporting Method" data. Draconian Mitigation Measures helped to contain pandemic to Hubei Province and Wuhan.

Figure 11: Predicted SOUTH KOREA CoVID-19 results. Pre-pandemic contact-tracing and large-scale CoVID-19 testing as Mitigation Measures have contained the pandemic.

Figure 12: Predicted ITALY CoVID-19 results. Additional curvature in the actual CoVID-19 data vs Model makes these predictions a likely worst-case.

Figure 13: Predicted GERMANY CoVID-19 results. This model gives a 
more gradual function for the Daily New CoVID-19 cases, making these predictions a likely worst-case.

Figure 14: Predicted SPAIN CoVID-19 results. This model gives a more gradual function for Daily New CoVID-19 cases, making these predictions a likely worst-case.

Figure 15: Predicted ECUADOR CoVID-19 results. Reports of chaos in Ecuador have been alarming. Poor CoVID-19 tracking and low testing may have skewed these results.

Figure 16: Predicted INDIA CoVID-19 results. Data shows only minimal mitigation at present. Further mitigations should help make these predictions a worst-case result.

Figure 17: Predicted ITALY CoVID-19 results, using an augmented 2parameter $\left\{\alpha_{S}, \beta_{S}\right\}$ Social Mitigation model. Total number of CoVID-19 cases is much less than Figure 12, but the model post-peak drop is much steeper, making this a likely best-case result.

\section{References}

1. https://www.nytimes.com/2020/03/11

/world/europe/coronavirus-merkel-germany.html

2. https://www.yahoo.com/news/recovered-coronavirus-patientstest-positive-161747102.html

3. https://www.scmp.com/news/hong-kong/health-environment /article/3075609/coronavirus-can-be-transmitted-through-faeces \{South China Morning Post/Hong Kong/Health and Environment\}

4. https://medium.com/@tomaspueyo /coronavirus-act-today-or-people-will-die-f4d3d9cd99ca; "Chart 2".

5. https://en.wikipedia.org/wiki

/2020_coronavirus_pandemic_in_South_Korea; "Timeline-March".

6. https://www.MedRxiv.org/ ID = $\overline{\mathrm{M}}$ EDRXIV/2020/043752v1, 03.25.2020,

"Forecasting COVID-19 impact on hospital bed-days, ICU-days, ventilator-days and deaths by US state in the next 4 months", IHME COVID-19 Health Service Utilization Forecasting Team.

7. https://www.bing.com/covid?form=CPVD07. 


\section{Early Stages of CoVID-19 Growth Outside of China Initial USA Data shows Doubling Time of $\sim 2.02$ Days}

(a) Total Cases of Coronavirus Outside of China

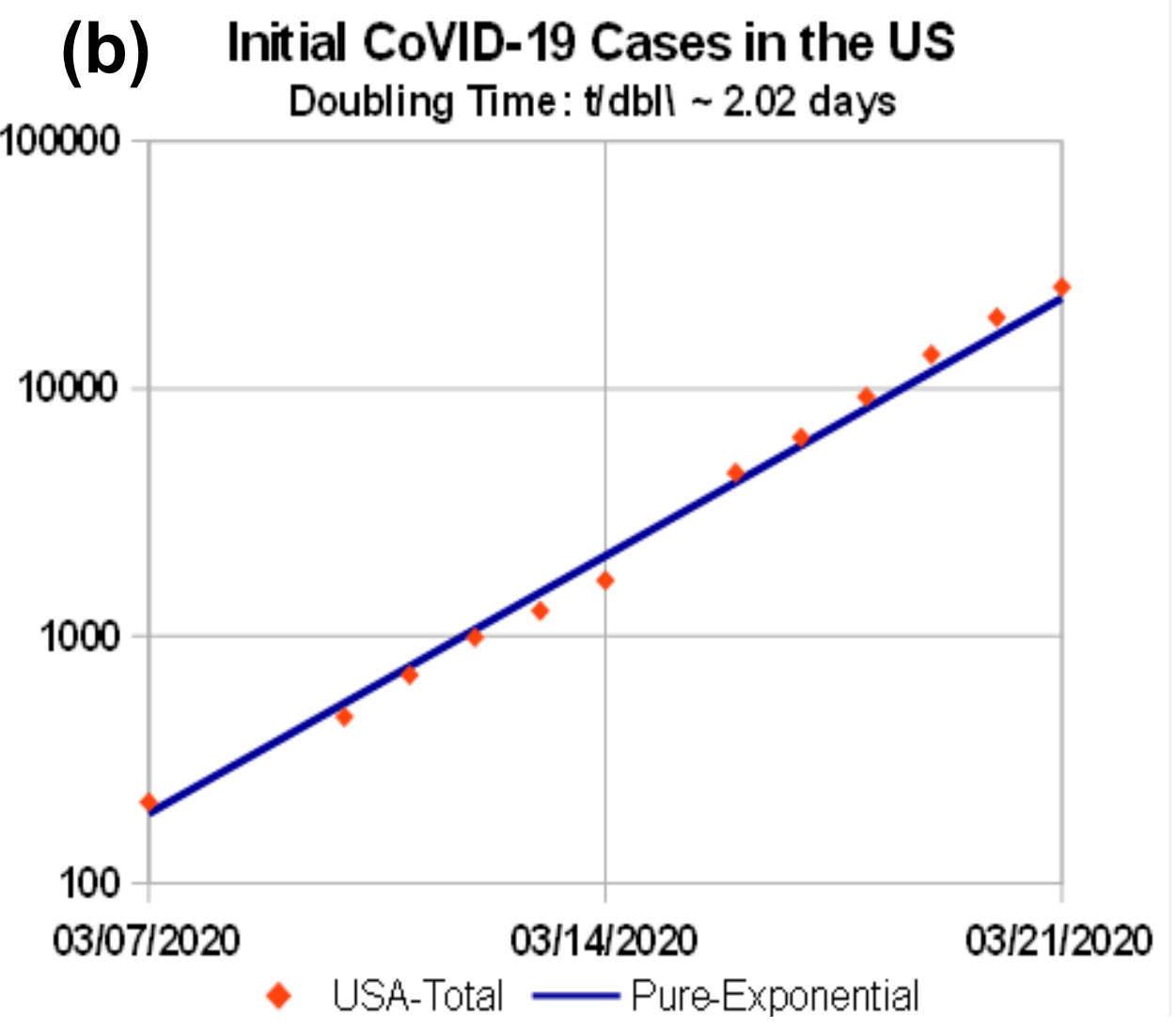

Sounce: Tomas Puejo, based on wondometers date

Left Side: Chart 2 from 3/11/2020 article "Coronavirus: Why You Must Act Now" by Thomas Pueyo https://medium.com/@ThomasPueyo/coronavirus-act-today-or-people-will-die-f4d3d9cd99ca Right Side: Initial Number of USA CoVID-19 Cases, Bing.com Coronavirus Daily Compilations

Figure 1: Early CoVID-19 Cases: (a) Outside of China, and (b) Just for the US. Both graphs show nearly exponential growth. 
Societal Mitigation Measures Slows Down CoVID-19 Growth

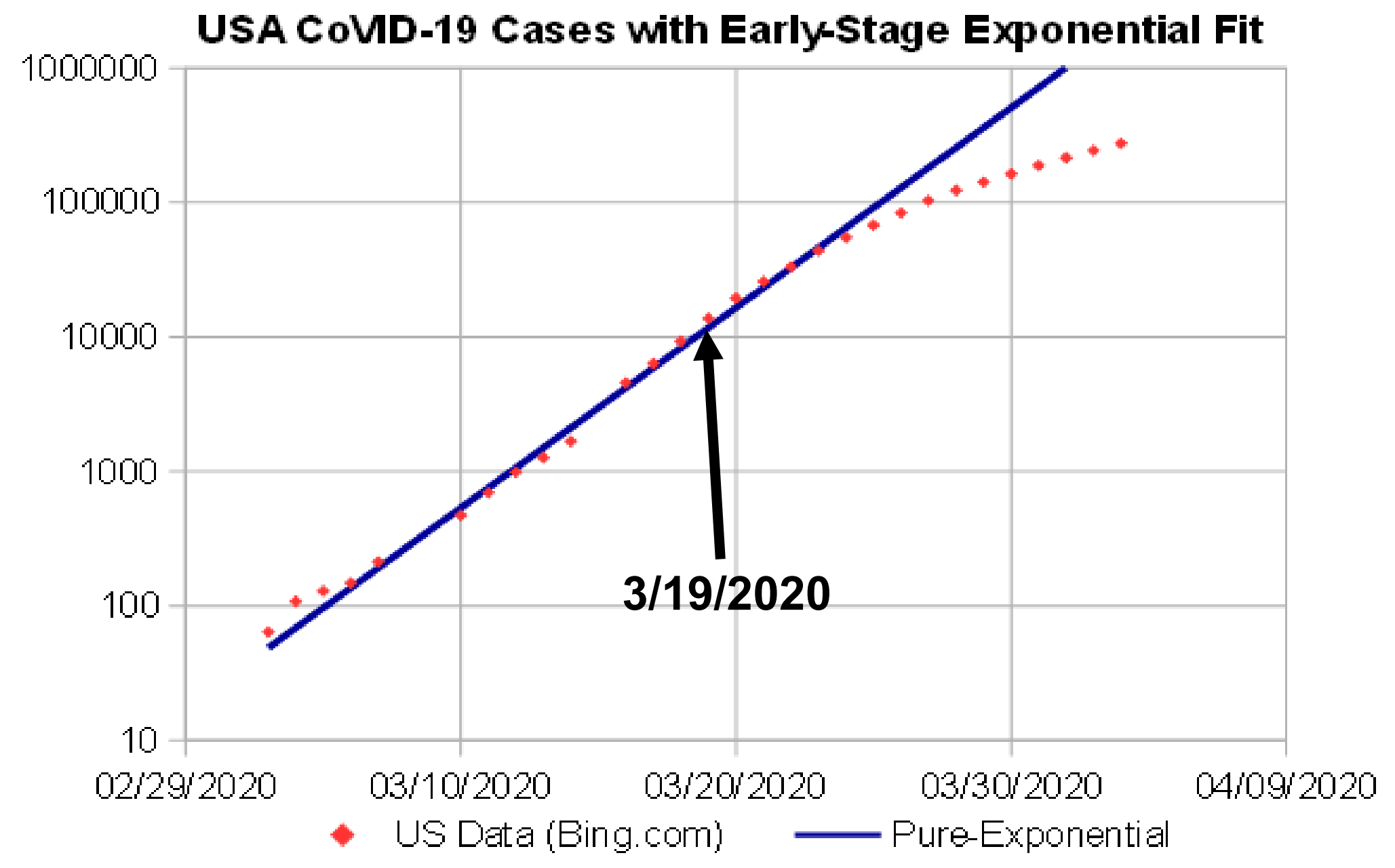

- 3/16-19/2020: CA Gov. Newsom Orders Closure of Schools followed by full lockdown of all Non-Essential Business; other states soon follow.

- CoVID-19 growth slows post-3/19/2020 Closures

Figure 2: USA CoVID-19 data, pre-vs-post mid-March 2020. Multi-State Mitigation Measures slowed growth, transitioning from straight-line to downward curvature. 
Model for USA CoVID-19 Pandemic, 4/19/20 Update: Peak= 4/19/2020

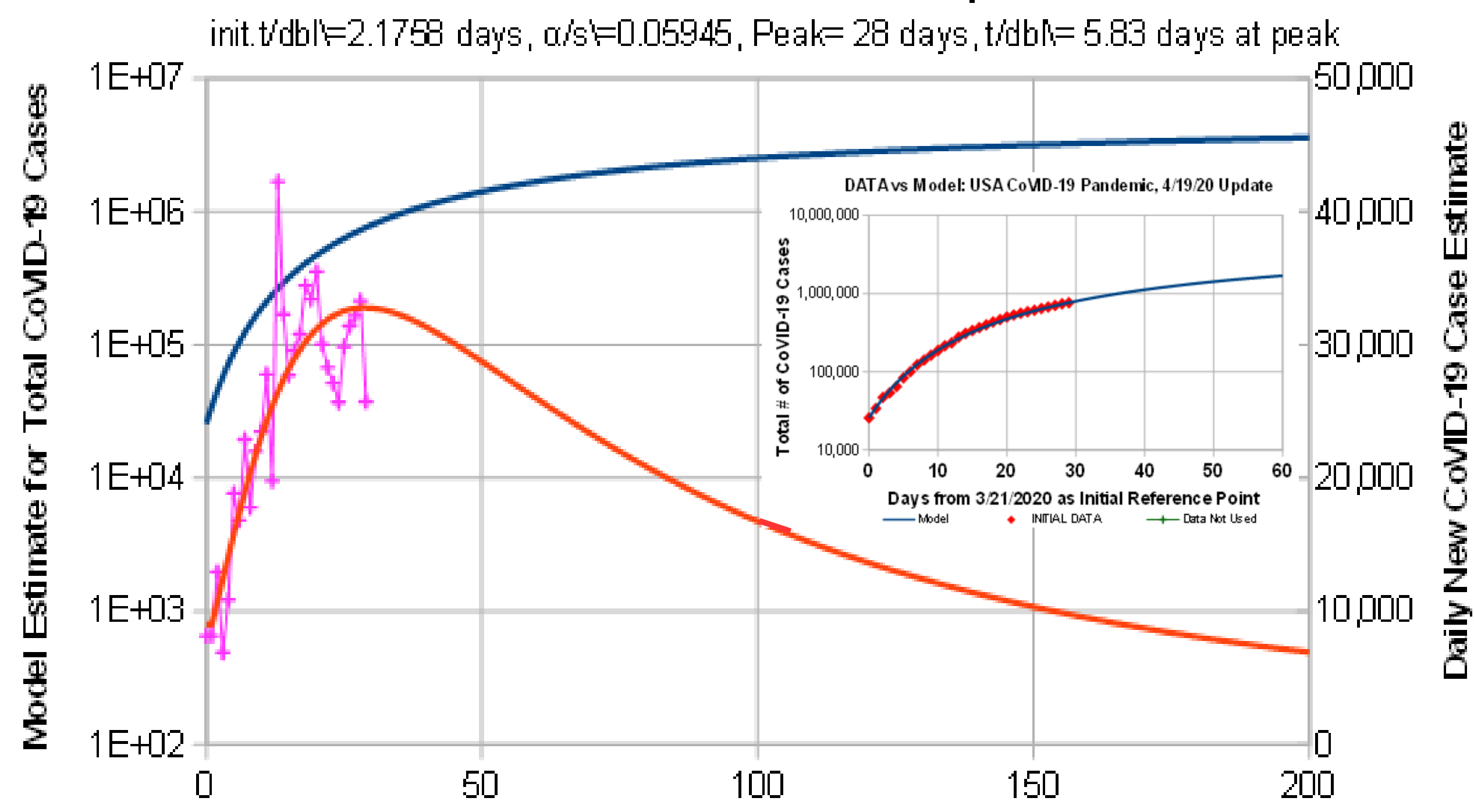

Days from 3/21/2020 Initial Reference Point, Predicted Max\#Cases $=5,464,000$ — Model _ Daily Nem Cases: Model +Daily New Cases: DATA

Figure 3: USA Model Predictions. To allow better mitigation predictions, only data after mid-March 2020 was included, when several Governors instituted mandatory lockdowns. 
Model for California CoVID-19 Pandemic, 4/17/20 Update: Peak= 6/07/2020

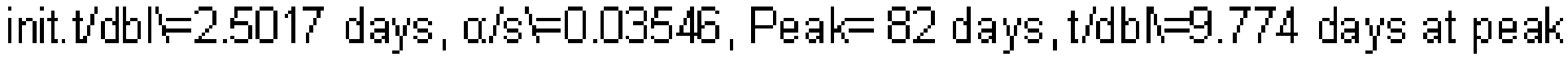

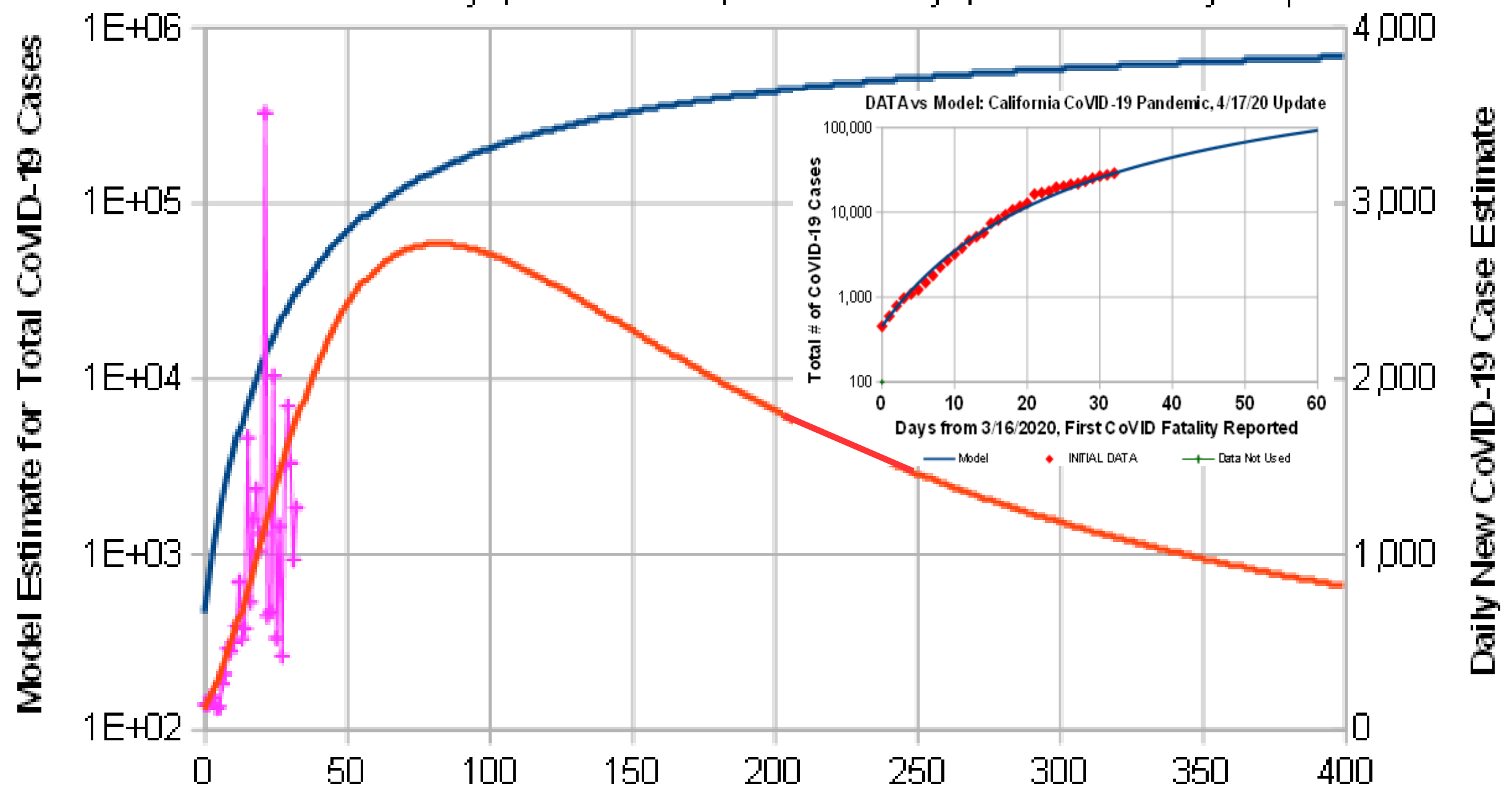

Days from $3 / 16 / 2020$, First CoVID Fat ality Reported, MaxtCases= 1,123,700
- Wodel
Daily New Cases: Model
+- Daily New Cases: DATA

Figure 4: Predicted California CoVID-19 results. After Gov. Gavin Newsom instituted widespread Mitigation Measures, projections showed significant improvement. 
Model for New York CoVID-19 Pandemic, 4/14/20 Update, Peak $=4 / 10 / 2020$

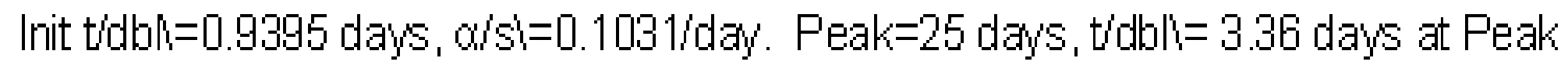

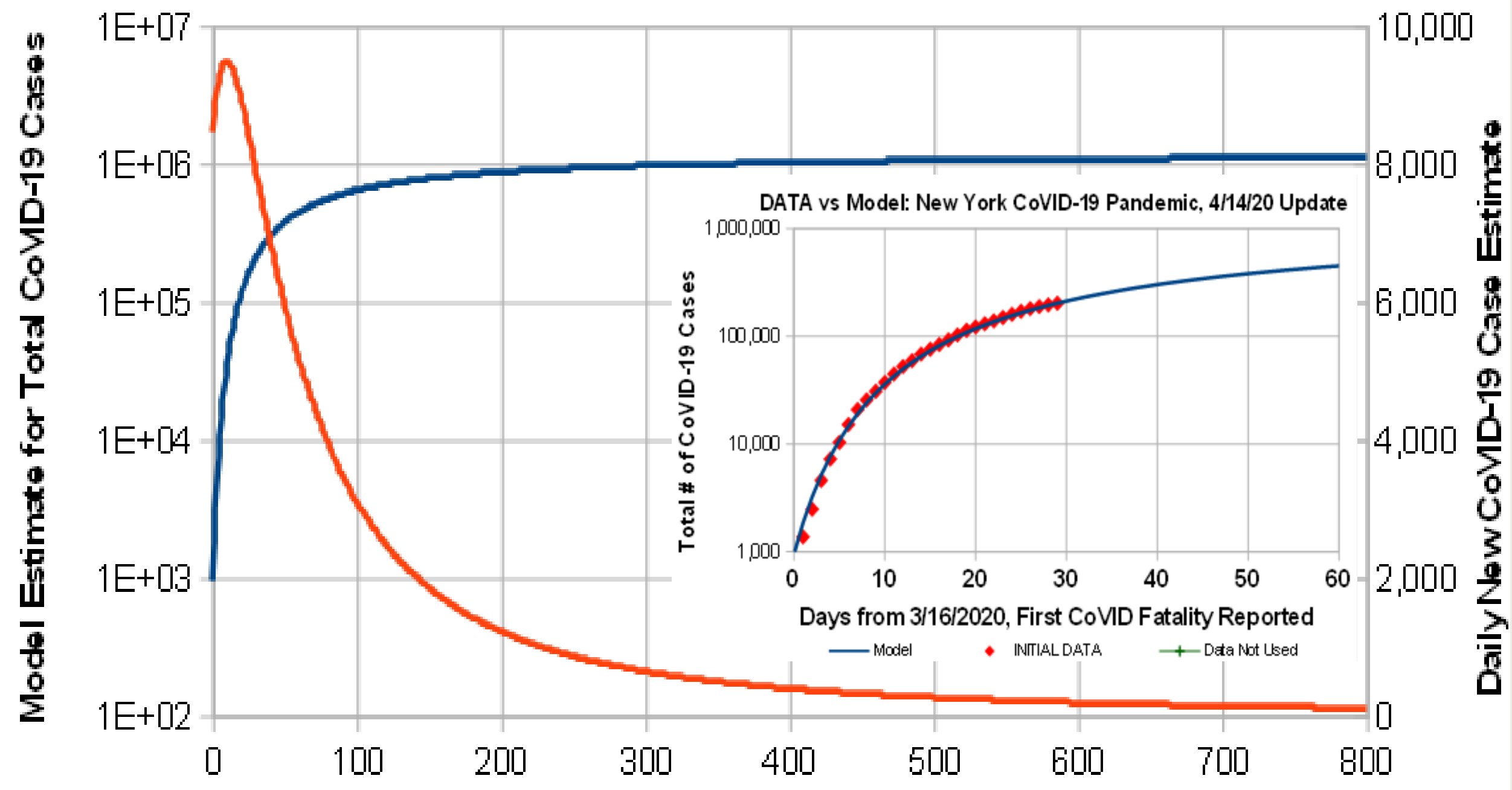

Days from 3/16/2020, First CoVID F atality Reported, Max\#-infected= 1,218,000 Model Daily New CoV/D-19 Cases

Figure 5: Predicted New York CoVID-19 results. A relatively high Mitigation Measure level and a short intrinsic doubling time creates a narrow spike in daily new cases. 
Model for WASHINGTON STATE CoVID-19 Pandemic

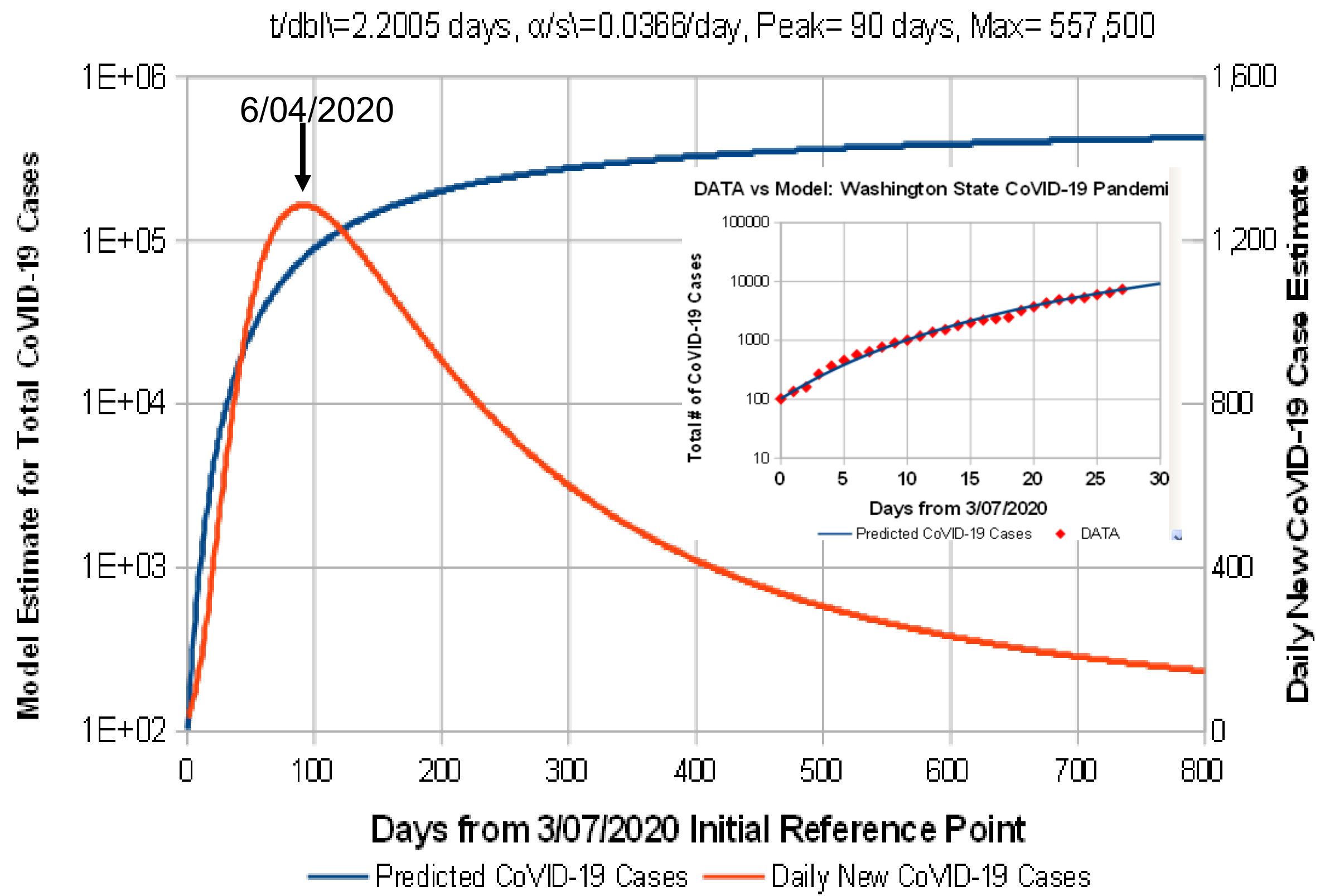

Figure 6: Predicted Washington State CoVID-19 results. The relatively low number of cases at Mitigation Measure start helps to give a relatively low final number of cases. 


\section{Model for ILLINOIS CoVID-19 Pandemic}

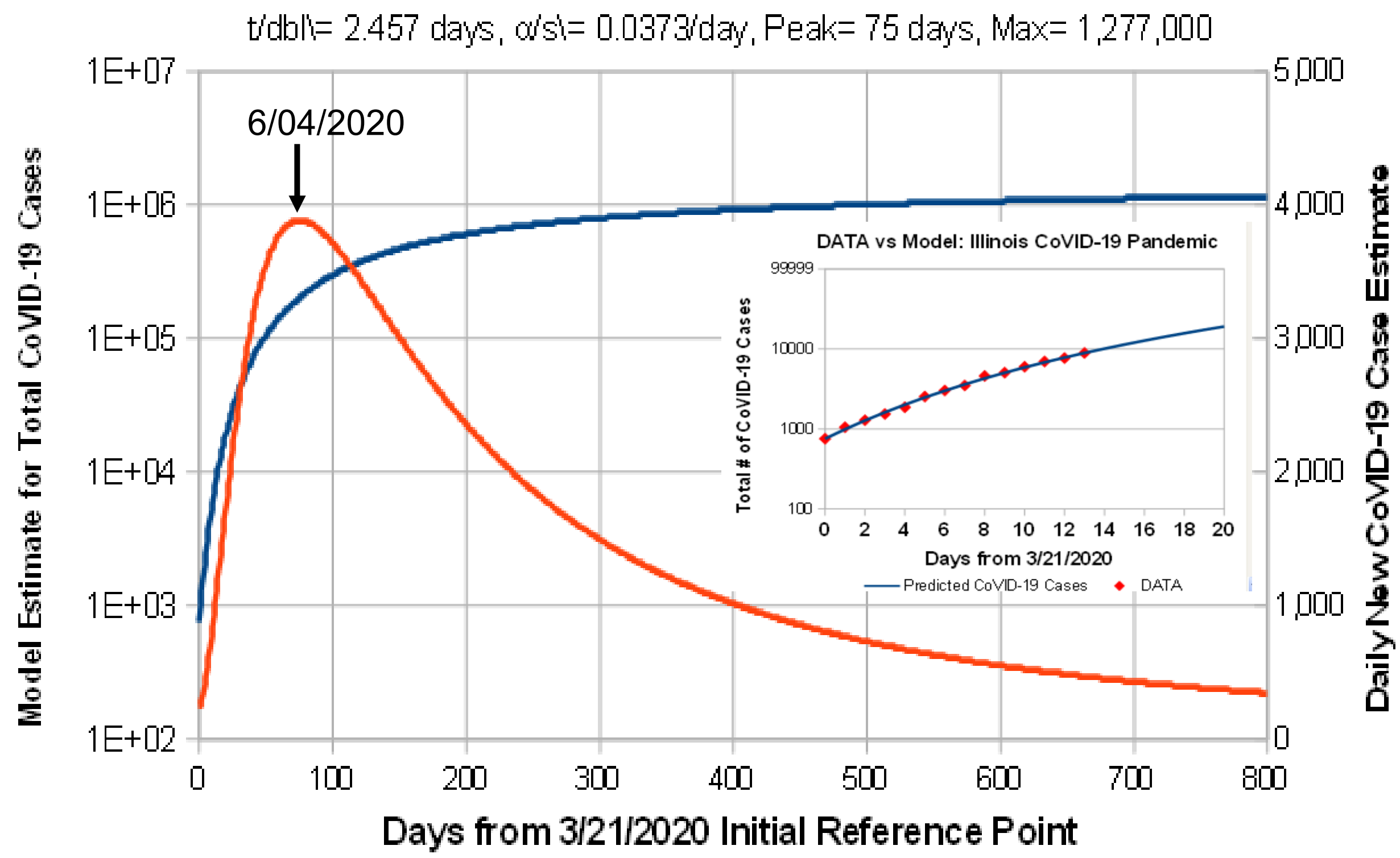

— Predicted CoVID-19 Cases — Daily New CoMD-19 Cases

Figure 7: Predicted Illinois CoVID-19 results. The slow doubling time and moderate amount of Mitigation Measures gives a slow increase to the predicted CoVID-19 peak. 


\section{Model for Florida CoVID-19 Pandemic}

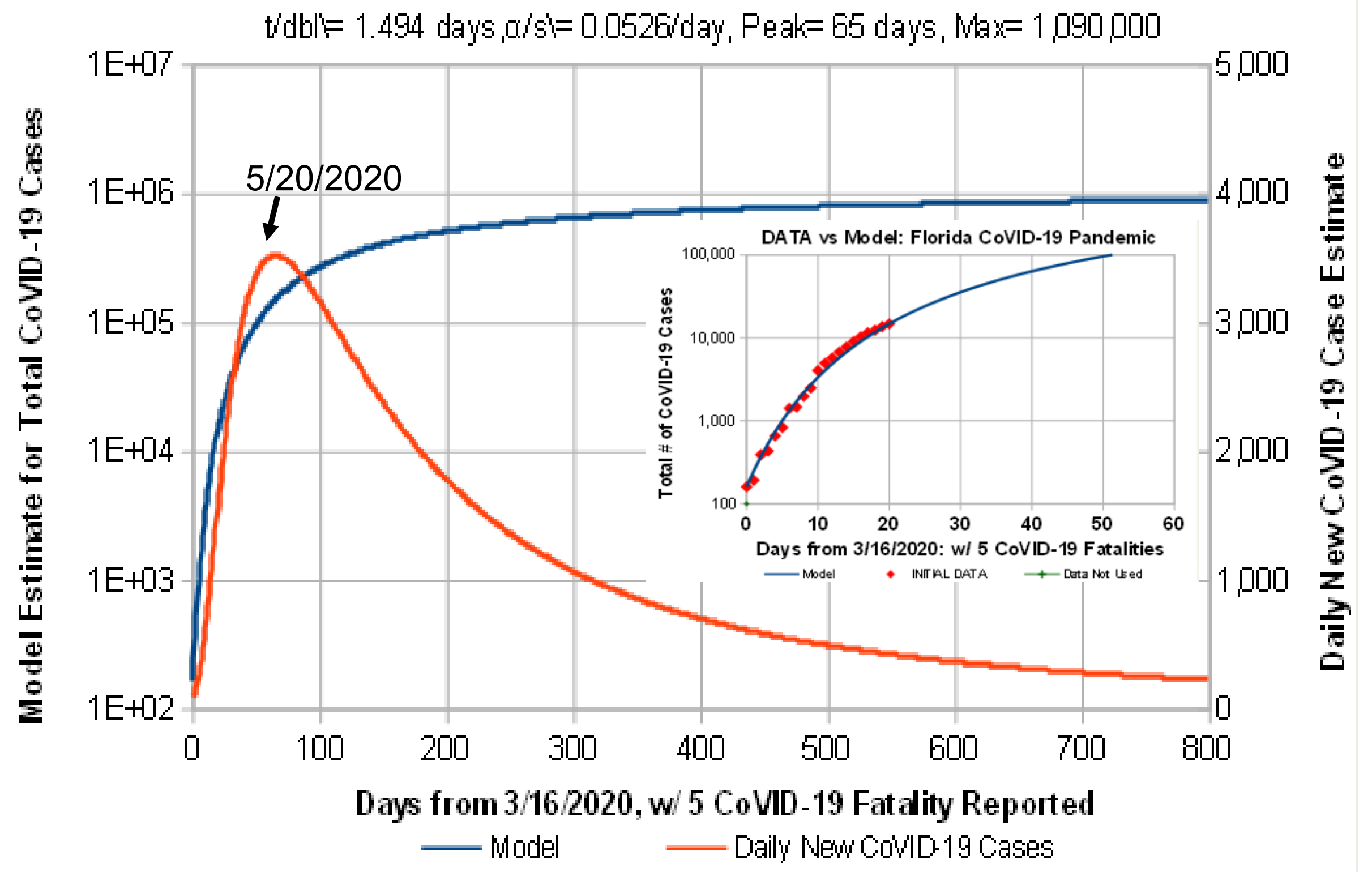

Figure 8: Predicted Florida CoVID-19 results. Many Florida counties instituted their own Mitigation Measures prior to a state-wide lockdown, substantially slowing CoVID-19 growth. 


\section{Model for WORLD CoVID-19 Pandemic}

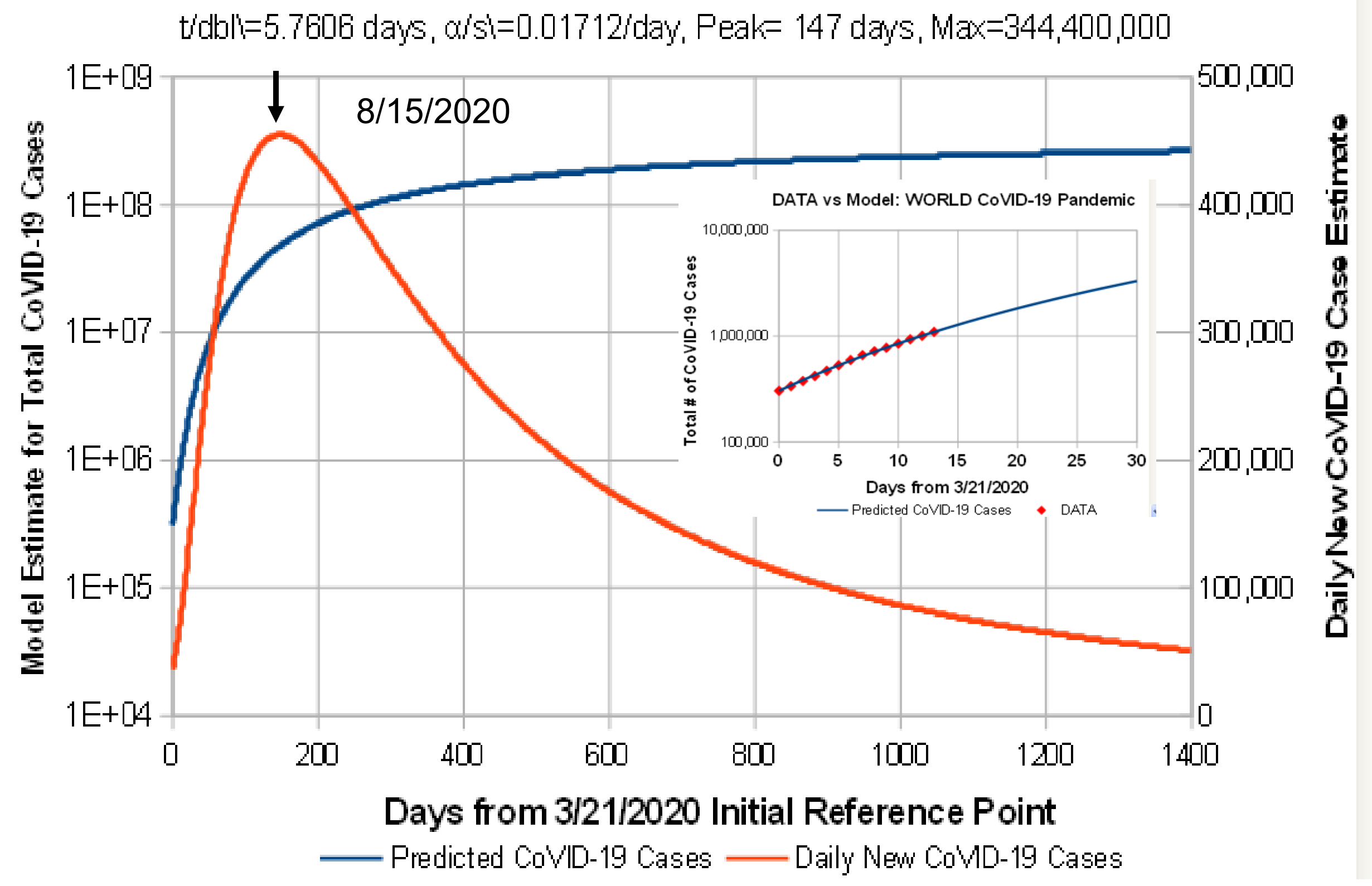

Figure 9: Model predictions for the WORLD, showing present-day low level of mitigation. 
Model for CHINA CoVID-19 Pandemic

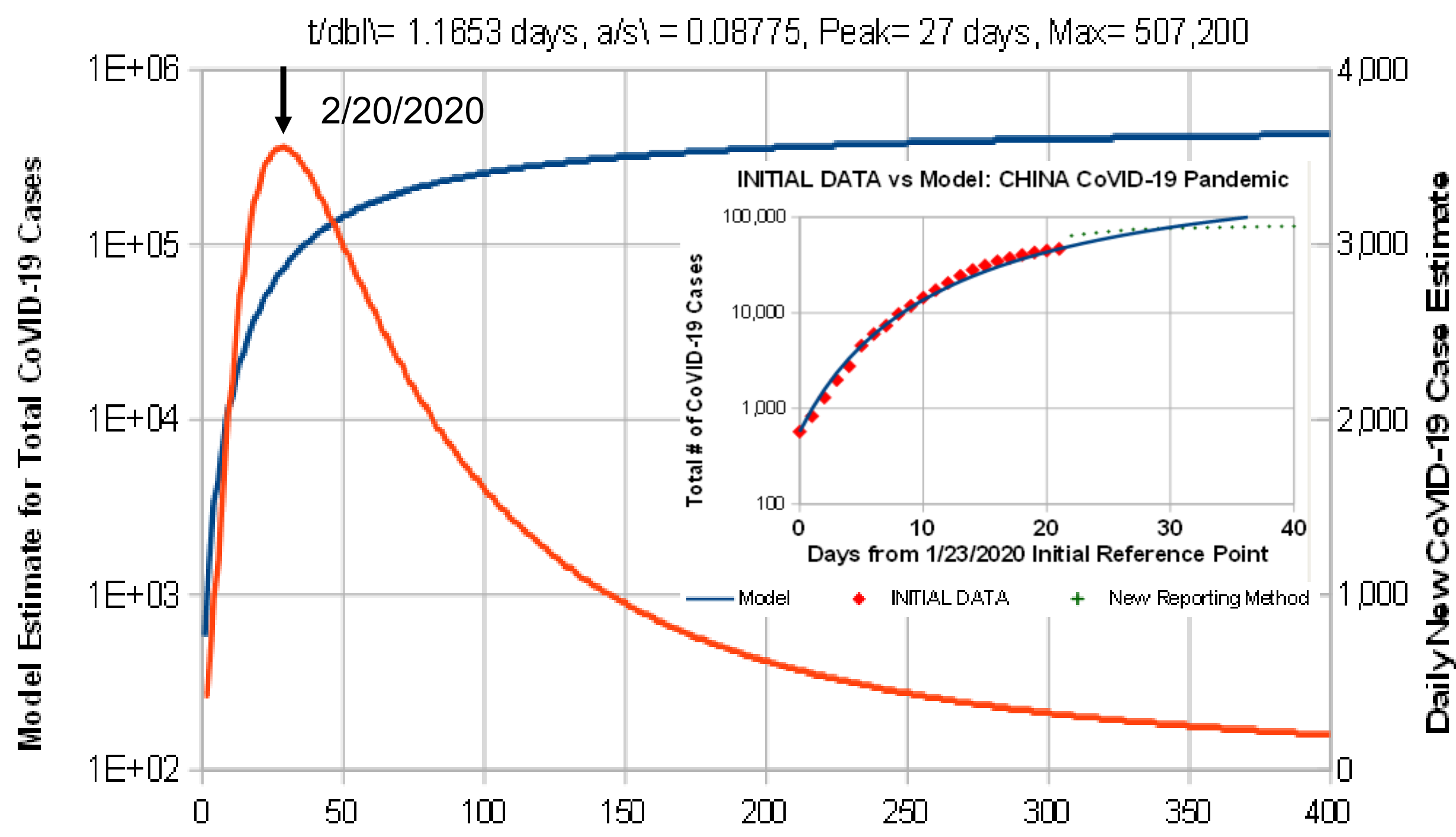

Days from 1/23/2020 Init.Ref.Point, Data to 2/13/20, prior to New Reporting Method

Figure 10: Predicted CHINA CoVID-19 results, using pre-"New Reporting Method" data. Draconian Mitigation Measures helped to contain pandemic to Hubei Province and Wuhan. 
Model for S-KOREA CoVID-19 Pandemic, Maximal Mitigation

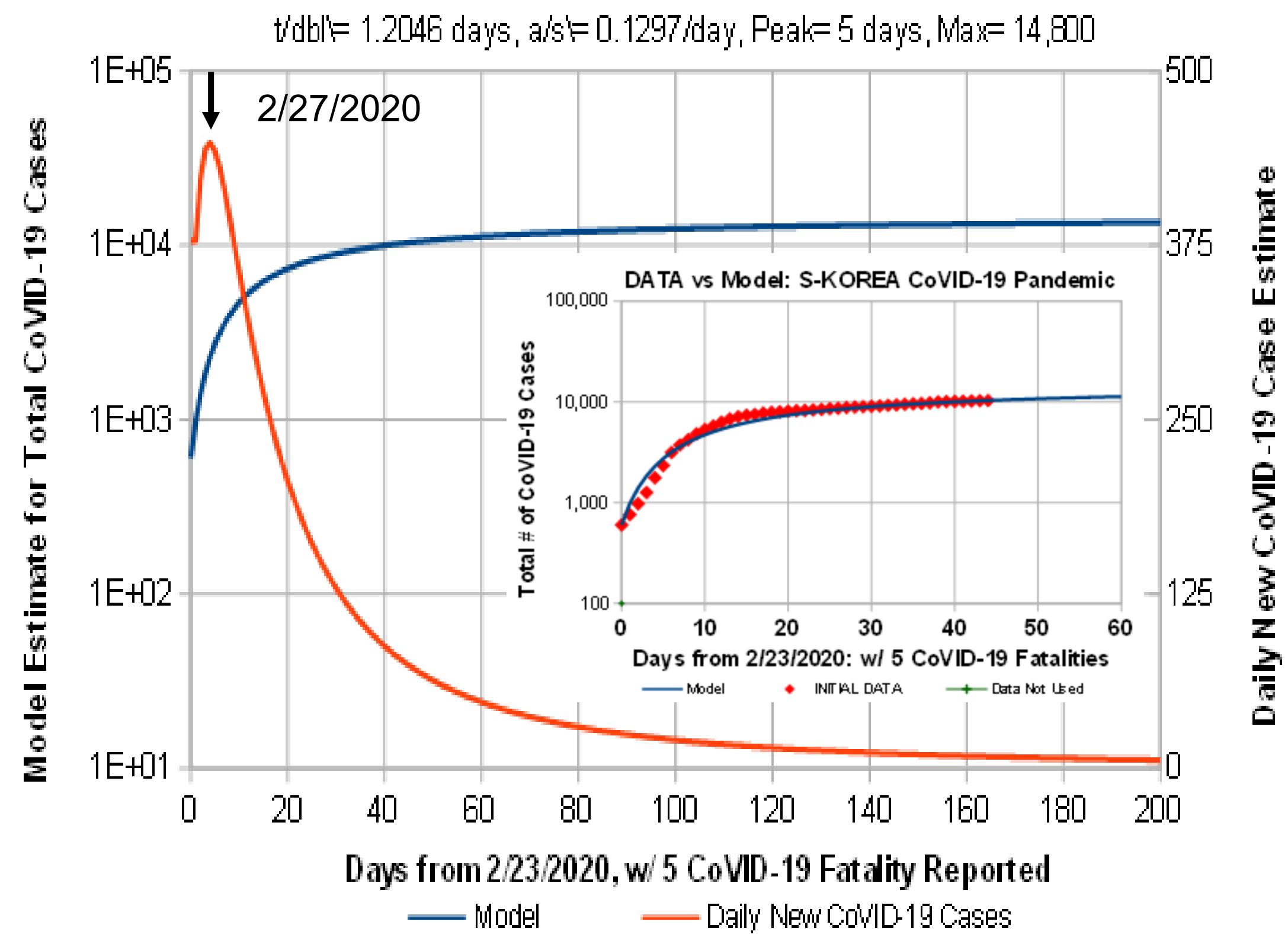

Figure 11: Predicted SOUTH KOREA CoVID-19 results. Pre-pandemic contact-tracing and large-scale CoVID-19 testing as Mitigation Measures have contained the pandemic. 
Model for ITALY CoVID-19 Pandemic, 4/18/20 Update: Peak= 4/29/2020

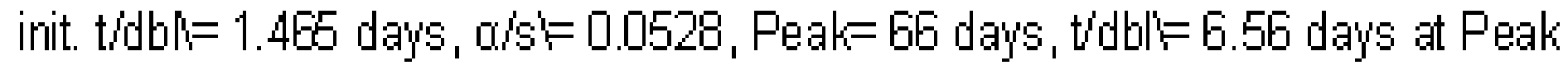

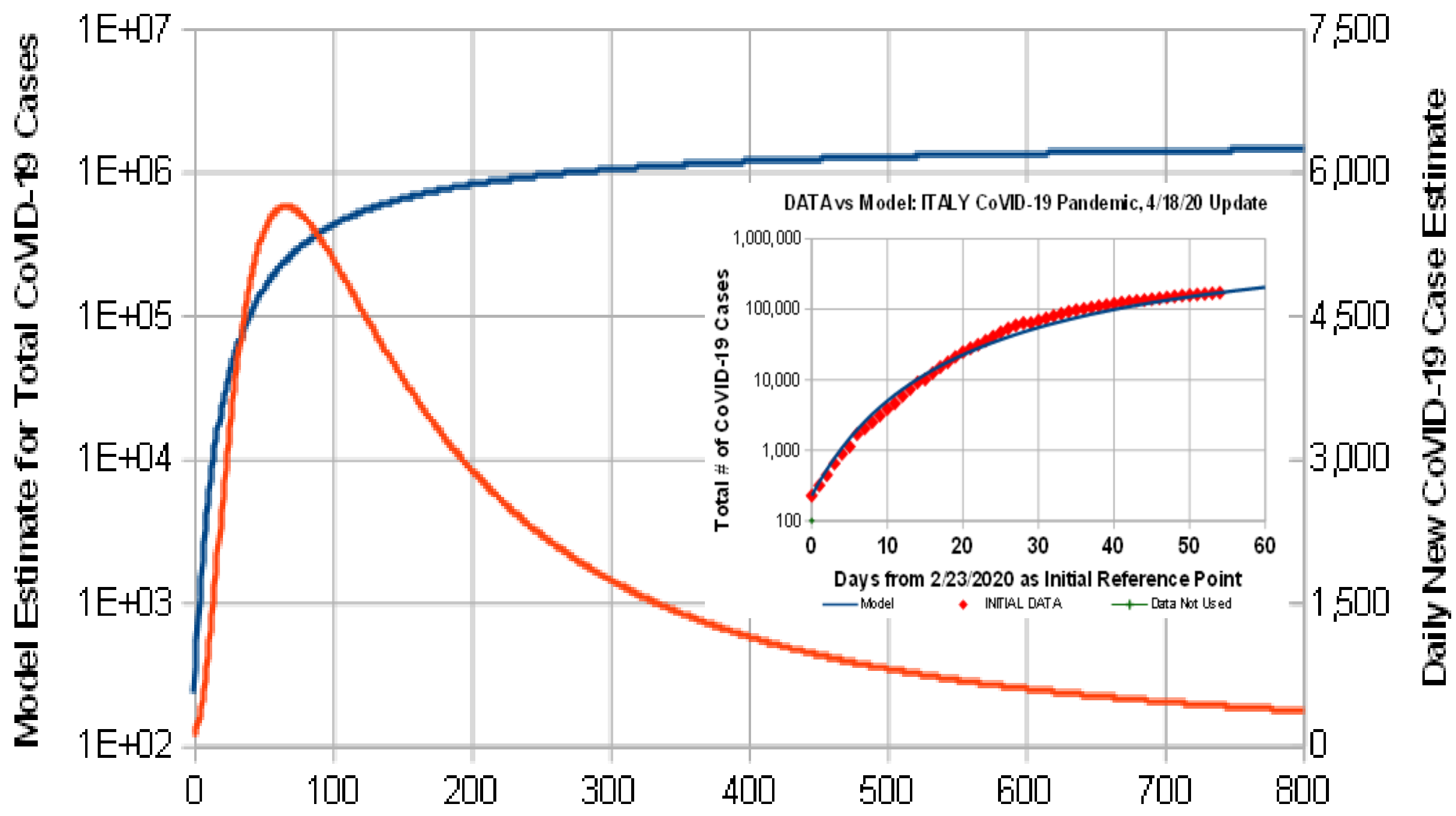

Days from 2/23/2020 Initial Reference Point, Predicted Max.\#Cases $=1,764,000$ 
Model for GERIMANY CoVID-19 Pandemic, 4/19/20 Update: Peak=4/08/2020

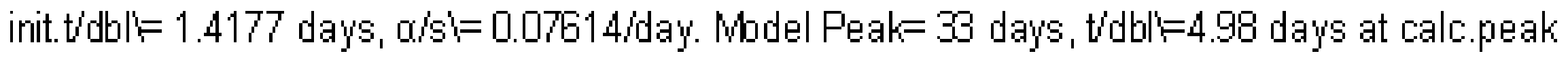

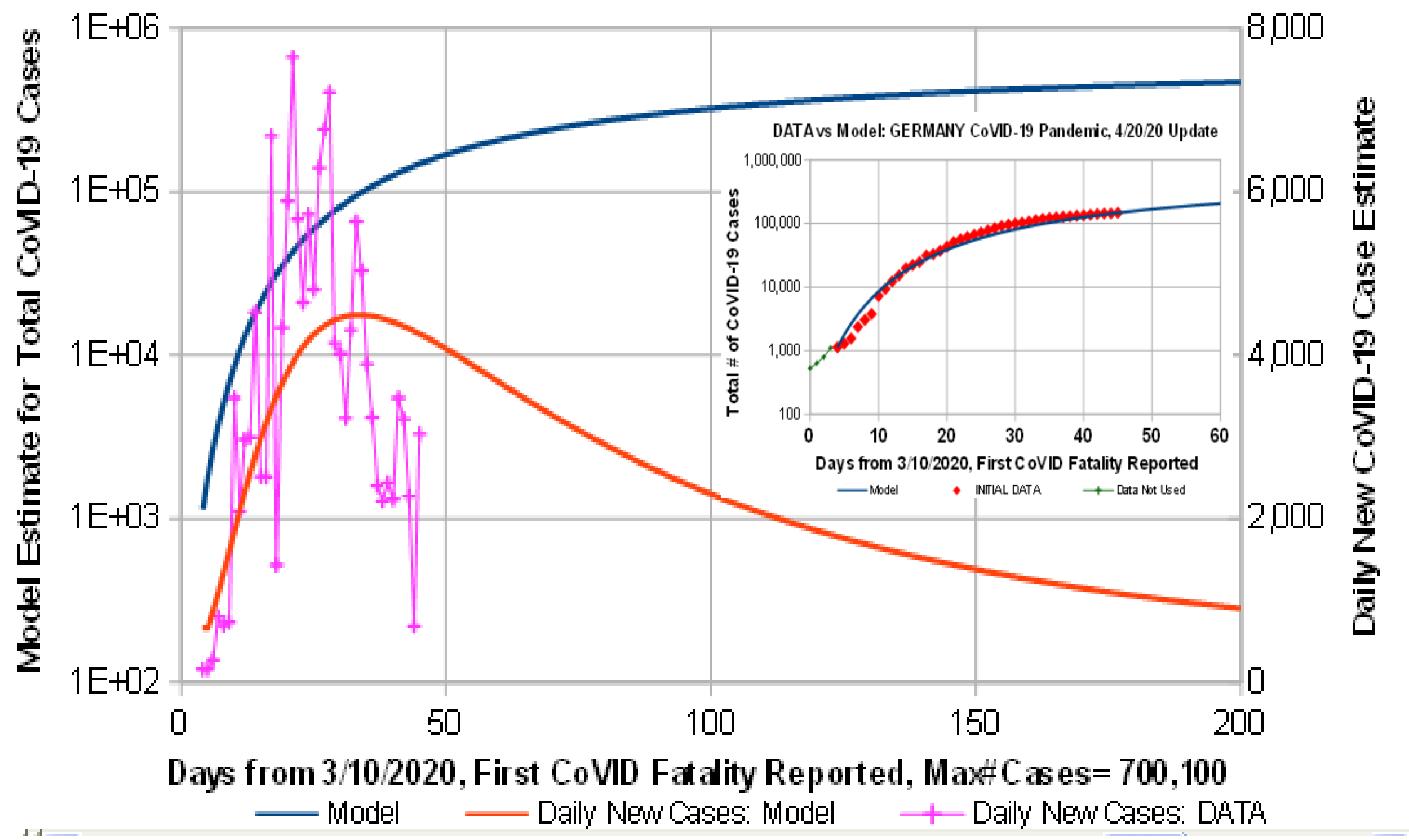

Figure 13: Predicted GERMANY CoVID-19 results. This model gives a more gradual function for the Daily New CoVID-19 cases, making these predictions a likely worst-case. 
Model for SPAIN CoVID-19 Pandemic, 4/19/20 Update: Peak=4/21/2020

init't/dbl'=1.1778 days, $\alpha / s^{\prime}=0.07058 / d a y$, Peak $=45$ days, $t / d b l=4.91$ days at model.peak

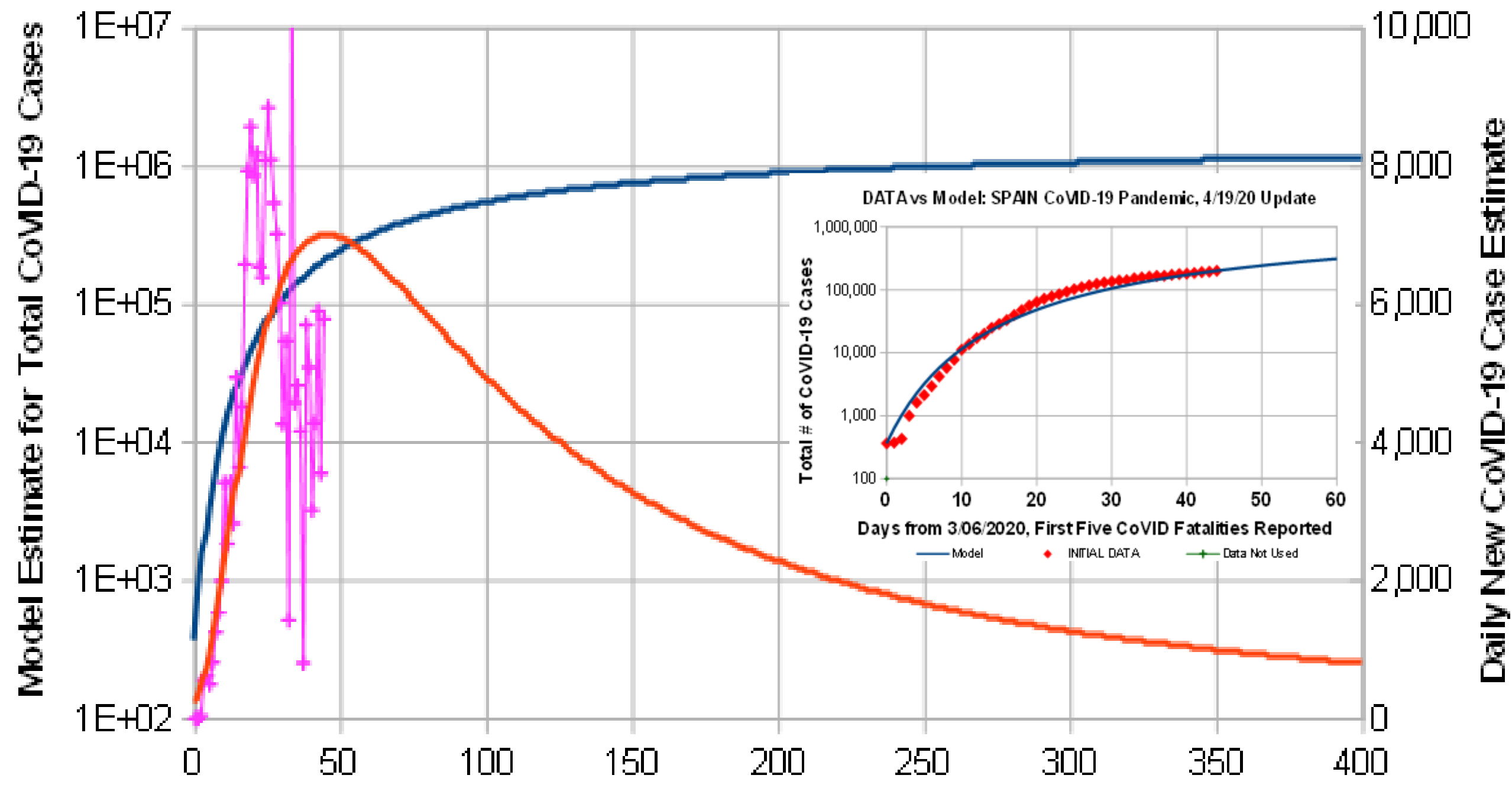

Days from 306/2020, First Five CoVID Fatalities Reported, Model.Max\#Cases $=1,526,000$ — Model —Daily New Cases: Model —Daily New Cases: DATA

Figure 14: Predicted SPAIN CoVID-19 results. This model gives a more gradual function for Daily New CoVID-19 cases, making these predictions a likely worst-case. 
Mo del for ECUADOR COVID-19 Pandemic

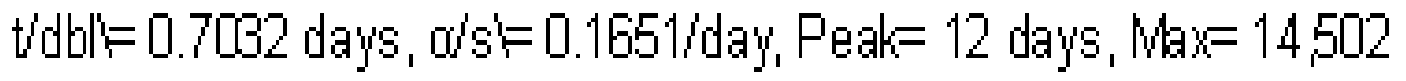

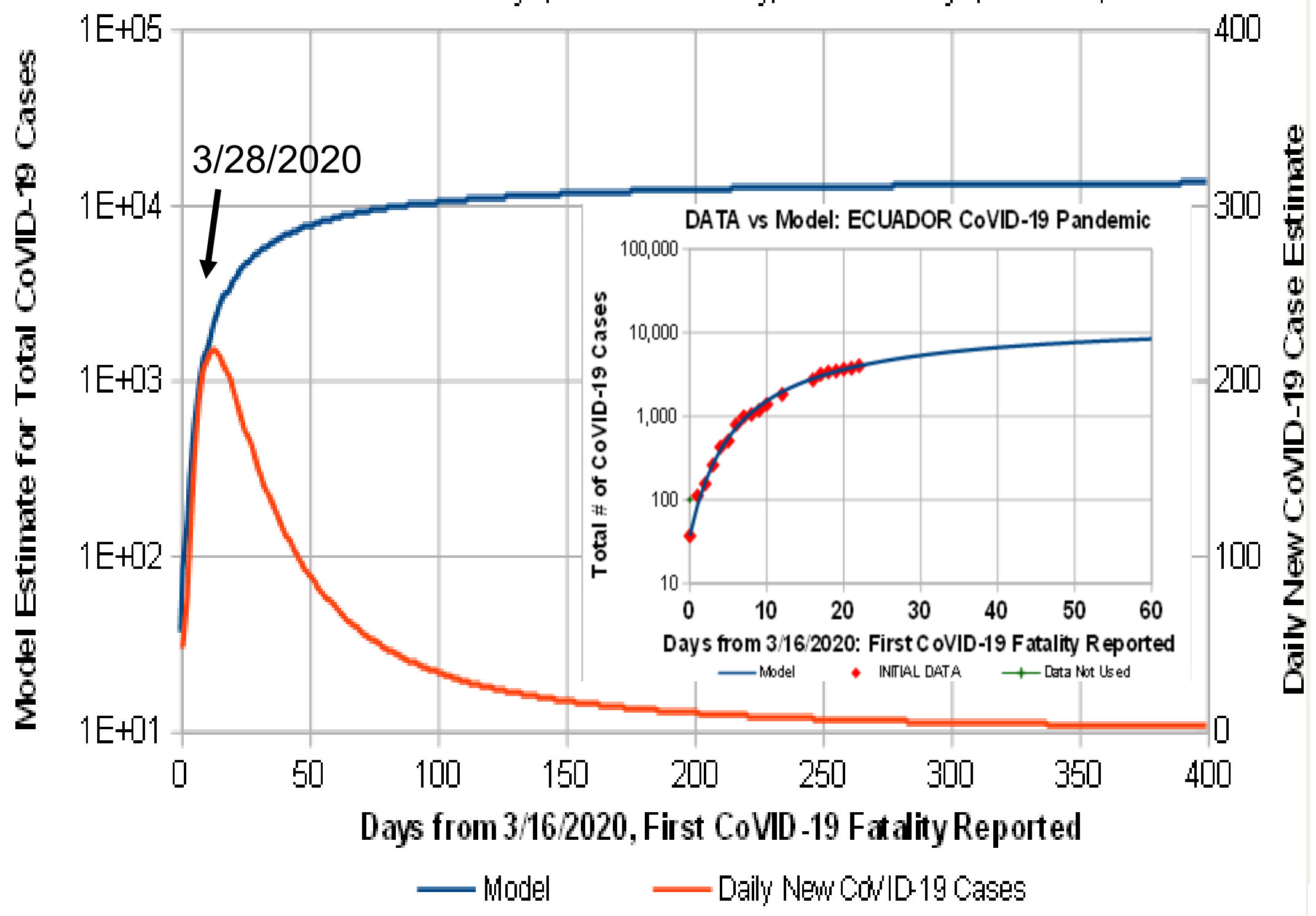

Figure 15: Predicted ECUADOR CoVID-19 results. Reports of chaos in Ecuador have been alarming. Poor CoVID-19 tracking and low testing may have skewed these results. 
Model for INDIA CoVID-19 Pandemic, 4/16/20 Update, Minimal Mitigation, 5/30/2021 Peak

init.t'db'=3.135 days, $\alpha s^{\prime}=0.0148 / d a y$, Peak= 441 days, $t^{\prime} d b \mid '=9.67$ days at Peak

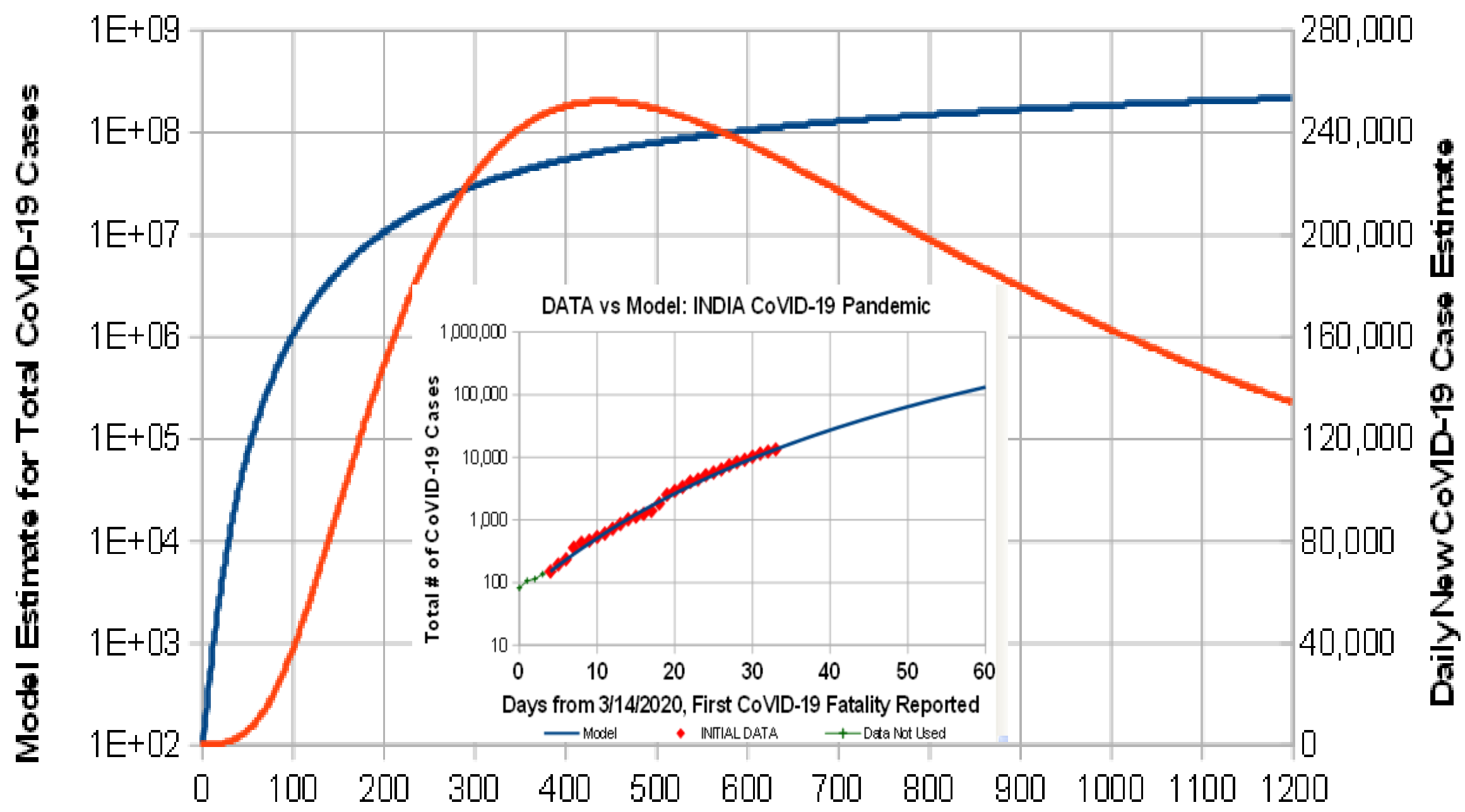

Days from 3/14/2020: 1st CoVID-19 Fatality Reported, Final 17.38\% Infection Rate Predicted

Figure 16: Predicted INDIA CoVID-19 results. Data shows only minimal mitigation at present. Further mitigations should help make these predictions a worst-case result. 
$\{K o, \alpha / s l, \beta / s \mid\}$ Augmented Model, ITALY CoVID-19 Pandemic, 4/18/20 Update: Peak= 3/29/2020

init. $t / d b l=2.56$ days, $\alpha / s t=.04583 / d a y, \beta / s t=-0.1725$, Peak $=36$ days, $t / d b l=4.13$ days at peak

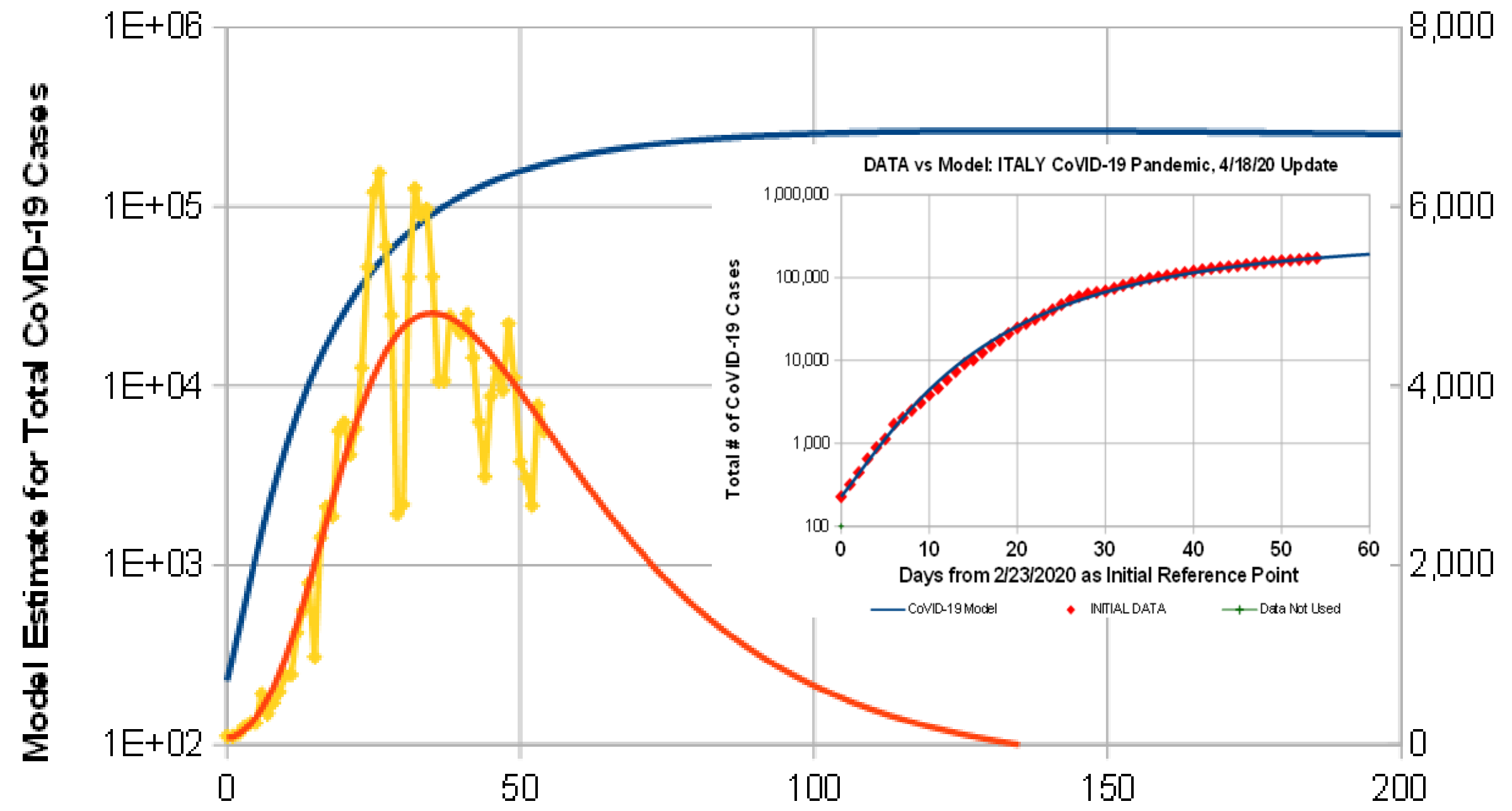

Days from 2/23/2020 Initial Reference Point, Predicted Max.\#C ases= 264,820

Figure 17: Predicted ITALY CoVID-19 results, using an augmented 2-parameter $\{\alpha / s|, \beta / s|\}$ Social Mitigation model. Total number of CoVID-19 cases is significantly less than Fig. 12, but the model post-peak drop is much steeper, making this a likely best-case result. 